\title{
Transcriptome-enabled marker discovery and mapping of plastochron-related genes in Petunia spp.
}

Yufang Guo ${ }^{\dagger}$, Krystle E. Wiegert-Rininger ${ }^{\dagger}$, Veronica A. Vallejo, Cornelius S. Barry and Ryan M. Warner ${ }^{*}$

\begin{abstract}
Background: Petunia (Petunia $\times$ hybrida), derived from a hybrid between $P$. axillaris and $P$. integrifolia, is one of the most economically important bedding plant crops and Petunia spp. serve as model systems for investigating the mechanisms underlying diverse mating systems and pollination syndromes. In addition, we have previously described genetic variation and quantitative trait loci (QTL) related to petunia development rate and morphology, which represent important breeding targets for the floriculture industry to improve crop production and performance. Despite the importance of petunia as a crop, the floriculture industry has been slow to adopt marker assisted selection to facilitate breeding strategies and there remains a limited availability of sequences and molecular markers from the genus compared to other economically important members of the Solanaceae family such as tomato, potato and pepper.

Results: Here we report the de novo assembly, annotation and characterization of transcriptomes from P. axillaris, P. exserta and P. integrifolia. Each transcriptome assembly was derived from five tissue libraries (callus, 3-week old seedlings, shoot apices, flowers of mixed developmental stages, and trichomes). A total of 74,573,54,913, and 104,739 assembled transcripts were recovered from P. axillaris, P. exserta and P. integrifolia, respectively and following removal of multiple isoforms, 32,994 P. axillaris, 30,225 P. exserta, and 33,540 P. integrifolia high quality representative transcripts were extracted for annotation and expression analysis. The transcriptome data was mined for single nucleotide polymorphisms (SNP) and simple sequence repeat (SSR) markers, yielding 89,007 high quality SNPs and 2949 SSRs, respectively. 15,701 SNPs were computationally converted into user-friendly cleaved amplified polymorphic sequence (CAPS) markers and a subset of SNP and CAPS markers were experimentally verified. CAPS markers developed from plastochron-related homologous transcripts from $P$. axillaris were mapped in an interspecific Petunia population and evaluated for co-localization with QTL for development rate.
\end{abstract}

Conclusions: The high quality of the three Petunia spp. transcriptomes coupled with the utility of the SNP data will serve as a resource for further exploration of genetic diversity within the genus and will facilitate efforts to develop genetic and physical maps to aid the identification of QTL associated with traits of interest.

Keywords: Petunia, Transcriptome sequencing, Molecular markers, Single nucleotide polymorphisms, Development rate, Floriculture

\footnotetext{
* Correspondence: warnerry@msu.edu

${ }^{\dagger}$ Equal contributors

Department of Horticulture, Michigan State University, East Lansing, MI 48824, USA
} 


\section{Background}

The genus Petunia resides within the Solanaceae family and contains 20 species and subspecies that are native to South America [1]. Petunia $\times$ hybrida (petunia) is an important ornamental crop plant and represents a hybrid species derived in the nineteenth century from a cross between $P$. axillaris and $P$. integrifolia [2]. Subsequent breeding has introgressed traits from additional Petunia spp. that, together with natural variation resulting from mutations in key genes, have contributed to the wide diversity in plant and floral morphology and flower color that exists within the pool of commercially available germplasm [2-7]. In cool climates in the Northern Hemisphere, petunia is often produced in greenhouses during the winter months for distribution to spring markets once it reaches an optimal size and begins to flower $[8,9]$. Therefore, a high percentage of the cost of crop production is related to energy consumption and growers are often faced with the dilemma of either reducing greenhouse temperatures, thereby extending the growing time of the crop and incurring increased labor costs, or elevating the growing temperature and increasing energy costs but reducing the duration of crop growth $[8,9]$. Thus, understanding the factors that impact crop timing traits may facilitate the selection of petunia varieties with an increased rate of vegetative node formation (development rate) at either optimal or suboptimal growing temperatures, or varieties that initiate flowering following emergence of fewer leaf nodes. We have previously documented that accessions of $P$. axillaris and $P$. integrifolia possess increased development rate when compared to a diverse pool of commercial petunia germplasm, suggesting genetic variation for this trait within the genus [10]. This was confirmed in an interspecific $\mathrm{F}_{2}$ population of a cross between $P$. axillaris and $P$. integrifolia that identified three quantitative trait loci (QTL) on chromosomes 1, 2 and 5 that affected development rate and explained $34 \%$ of the observed variation [11]. The molecular basis underlying these QTL remains to be identified.

The genetic determinants of development rate, often referred to as plastochron, are multifaceted, complex and not fully understood but are, at least in part, linked to hormonal control of meristem size and activity. For example, transgenic tobacco (Nicotiana tabacum) plants with increased cytokinin oxidase activity and a concomitant reduction in cytokinin levels displayed reduced meristem size and delayed plastochron when compared to wild type [12]. Similarly, characterization of Arabidopsis mutants with reduced auxin levels and disrupted auxin transport also influence plastochron [13-15]. In addition, mutations at the plastochron 1 and plastochron 2 loci of rice, which encode a cytochrome P450 of unknown function and a MEI2-like RNA binding protein homolog, respectively, also influence development rate but do so independently of each other [16, 17]. In Arabidopsis, the SQUAMOSA PROMOTER BINDING PROTEIN-LIKE (SPL) transcription factors SPL9 and $S P L 15$ act redundantly to influence plastochron and over-expression of miR156, which targets multiple SPLs, shortens plastochron [18]. Analogous to the relationship of the plastochron 1 and 2 loci of rice [17], the SPL/miR156 regulatory module acts independently of CYP78A5/KLUH, which is a putative ortholog of rice plastochron 1 [18]. The involvement of the miRNA pathway in influencing plastochron is further supported by the characterization of the serrate and altered meristem program (amp1) mutants of Arabidopsis, which display reduced and increased rates of leaf initiation, respectively [19-22]. SERRATE encodes a zinc finger protein required for miRNA biogenesis and RNA splicing while AMP1 associates with ARGONAUTE1 at the endoplasmic reticulum and is required for translation inhibition through the exclusion of miRNA target mRNAs from polysomes [19, 20, 23]. Furthermore, mutations in AMP1 homologs in maize and rice confer similar pleiotropic phenotypes to those exhibited by Arabidopsis amp1 mutants, including altered plastochron [24, 25]. Together, these data suggest complex regulation of plastochron that involves different regulatory modules, including hormone and miRNA pathways.

The development of next generation sequencing technology has revolutionized biology and in particular, transcriptome sequencing provides a cost effective strategy for generating sequence and expression information from the gene space of non-model organisms or from species with large complex genomes [26, 27]. In plants, transcriptome sequencing has facilitated gene discovery, the development of molecular markers and large scale analyses of genetic variation [28-33]. Despite the economic and biological importance of petunia, genomic information and molecular marker resources for this genus are limited [11,34-38], single nucleotide polymorphism (SNP) markers are currently unavailable and marker assisted selection is rarely utilized. In addition, although transcriptome resources are available for petunia, they are not extensive and most often are derived from the cultivated species $P$. hybrida or from specialized tissue types [39-41]. Herein, de novo assembly of reference transcriptomes of $P$. axillaris, $P$. integrifolia and $P$. exserta, derived from paired-end RNAseq analysis of five diverse tissues (callus, seedling, shoot apices, flowers and trichomes) is reported. Tissue types were selected to attempt to maximize the number of transcripts recovered while generating resources for studying traits of interest related to development and metabolism. These resources were utilized to develop a set of SNP, cleaved amplified polymorphic sequence (CAPS) and 
simple sequence repeat (SSR) markers that will facilitate QTL mapping and gene discovery for multiple traits within the genus, including those associated with development rate.

\section{Results and discussion}

Transcriptome assembly and annotation

Transcriptome sequencing of five tissue libraries, including callus, flowers, shoot apex, seedlings, and trichomes, from $P$. axillaris, $P$. exserta, and $P$. integrifolia yielded between $\sim 248$ and 294 M 100 nt reads, of which greater than $94 \%$ passed quality and trimming filters (Table 1 ). A two-step de novo assembly strategy (see Additional file 1: Figure S1) modified from [42] resulted in 74,573, 54,913 , and 104,739 transcripts $\geq 500$ bp respectively, for $P$. axillaris, $P$. exserta, and $P$. integrifolia. The two-step assembly strategy was employed to eliminate redundant reads in the flower and trichome libraries already present in the callus, shoot apex, and seedling libraries to aid the quality of the assembly.

The N50 value for each assembly was greater than 1950 and average transcript sizes of 1714 for $P$. axillaris, 1624 for $P$. exserta, and 1646 for $P$. integrifolia were obtained. The distribution of transcript sizes follows the same trend in each of the three species with the largest number of transcripts falling within the size bins of 500-1000 bp, and between 1001 and 1500 bp (Fig. 1a). CEGMA analysis [43] revealed that full length copies of

Table 1 Description of Petunia spp. tissues, libraries, and RNA-Seq data

\begin{tabular}{|c|c|c|c|c|}
\hline Species & Tissue & Library & $\begin{array}{l}\text { Number of raw } \\
\text { reads (millions) }\end{array}$ & $\begin{array}{l}\text { Number of filtered } \\
\text { reads (millions) }\end{array}$ \\
\hline \multirow[t]{5}{*}{ P. axillaris } & Callus & $A C$ & 57.0 & $54.6(95.8 \%)$ \\
\hline & Flower & $\mathrm{AF}$ & 54.3 & $52.3(96.2 \%)$ \\
\hline & $\begin{array}{l}\text { Shoot } \\
\text { Apex }\end{array}$ & $\mathrm{AA}$ & 70.4 & 67.9 (96.3 \%) \\
\hline & Seedling & AS & 65.3 & $62.8(96.0 \%)$ \\
\hline & Trichome & AT & 47.2 & 44.6 (94.5 \%) \\
\hline \multirow[t]{5}{*}{ P. exserta } & Callus & $\mathrm{EC}$ & 36.8 & 34.8 (94.5 \%) \\
\hline & Flower & EF & 66.7 & $63.0(94.4 \%)$ \\
\hline & $\begin{array}{l}\text { Shoot } \\
\text { Apex }\end{array}$ & EA & 56.6 & 53.6 (94.7 \%) \\
\hline & Seedling & ES & 43.9 & 41.9 (95.3 \%) \\
\hline & Trichome & ET & 45.0 & 41.6 (94.5 \%) \\
\hline \multirow{5}{*}{$\begin{array}{l}\text { P. } \\
\text { integrifolia }\end{array}$} & Callus & IC & 57.9 & 54.7 (94.5 \%) \\
\hline & Flower & IF & 64.4 & $61.2(95.0 \%)$ \\
\hline & $\begin{array}{l}\text { Shoot } \\
\text { Apex }\end{array}$ & IA & 61.4 & $58.2(94.8 \%)$ \\
\hline & Seedling & IS & 50.2 & 47.5 (94.7 \%) \\
\hline & Trichome & IT & 47.9 & 45.7 (95.4 \%) \\
\hline
\end{tabular}

${ }^{a}$ The number and percentage of total raw reads included in each transcriptome assembly after quality filters were applied
$>91 \%$ of the highly conserved eukaryotic genes are present in each of the transcriptome assemblies while partial sequences are present for almost $100 \%$ of these genes (Fig. 1b). These data are similar to those reported for other plant transcriptome assemblies [44, 45]. All high quality raw reads from each of the five libraries were also mapped back to their respective assembly with Bowtie and Tophat [46] generating mapping rates of $87.2 \%$ for P. axillaris, $86.8 \%$ for P. exserta, and $78.3 \%$ for P. integrifolia.

For $P$. axillaris and $P$. exserta, the majority of representative transcripts comprised a single isoform whereas approximately $60 \%$ of $P$. integrifolia transcripts possessed multiple isoforms (Table 2; Additional file 1: Figure S2). Where multiple isoforms were identified, the median number was two for $P$. axillaris and $P$. exserta, and three for $P$. integrifolia. The longest isoform was selected as the representative transcript yielding a total of 32,994, 30,225 and 33,540 representative transcripts in P. axillaris, $P$. exserta, and $P$. integrifolia, respectively. These transcripts covered between $\sim 47.5$ and $53.1 \mathrm{Mbp}$ of the transcriptome space (Table 2). The increased number of transcripts retrieved from the $P$. integrifolia assembly together with a higher number of transcripts with multiple isoforms, is likely the result of widespread heterozygosity within this species due to self-incompatibility [47].

Representative transcripts from each assembly were annotated using BLASTX searches against five publically available databases (Additional file 2: Dataset S1). Adopting quality thresholds of $\geq 30 \%$ coverage and $\geq 70 \%$ identity resulted in an annotation rate of approximately 70 \% (Fig. 1c), with the largest number of annotations retrieved from the RefSeq and NCBI nonredundant databases. Similar rates of annotation were reported for transcriptome assemblies of chickpea and red clover $[48,49]$. In addition, of the 1944 predicted $\mathrm{Pe}$ tunia proteins available in GenBank, $89 \%$ are present in the $P$. axillaris, $P$. exserta, and $P$. integrifolia transcriptome assemblies.

Open reading frames (ORFs) were extracted from the representative transcripts and the predicted protein sequences were searched for orthologous gene clusters using OrthoMCL [50]. Among all comparisons, approximately 21,000 orthologous clusters were identified, with $P$. axillaris, $P$. exserta, and $P$. integrifolia sharing 13,747 clusters (Fig. 2; Additional file 3: Dataset S2). A larger number of orthologous clusters were found between $P$. axillaris and $P$. exserta, likely due to their closer phylogenetic relationship [1]. While $P$. integrifolia was found to house over double the number of unique clusters (Fig. 2; Supplemental Dataset 2). In addition, Gene Ontology (GO) annotations (Additional file 4: Dataset S3) of the representative transcripts showed highly equivalent representation of biological process categories 

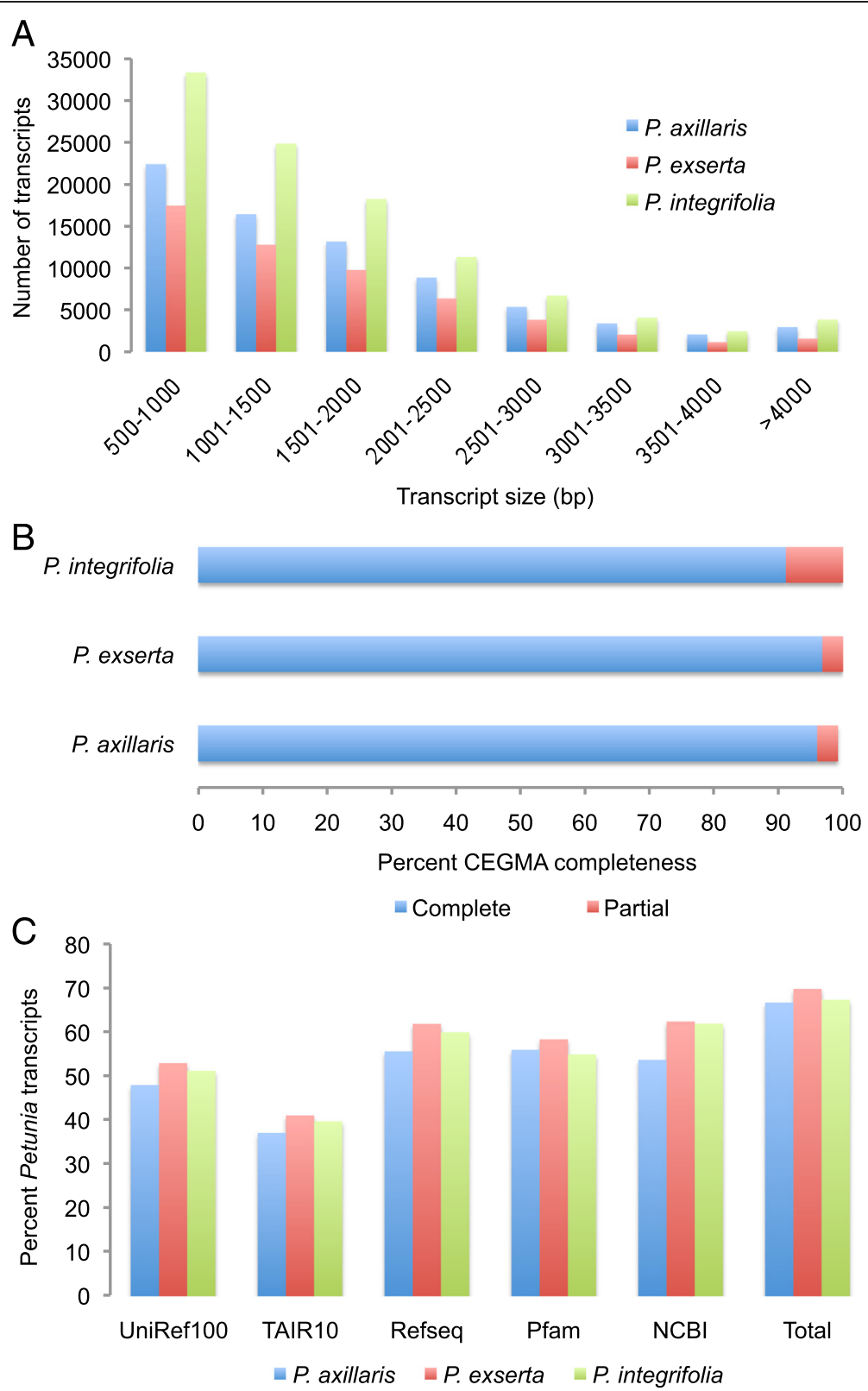

Fig. 1 Quality metrics of the Petunia spp. transcriptome assemblies. a Size distribution of assembled transcripts. b CEGMA completeness assessment of the transcriptome assemblies. c Percentage of P. axillaris, P. exserta, and P. integrifolia unigenes with assigned functional annotations from UniRef100, TAIR10, RefSeq, the Pfam domain database, and NCBI GenBank non-redundant protein set. In addition, the total percentage of annotated unigenes per species is presented

Table 2 Total number of de novo assembled transcripts in each of the Petunia axillaris, P. exserta, and P. integrifolia transcriptomes in relation to the number of representative transcripts and their total coverage of the transcriptome space

\begin{tabular}{lllllll}
\hline & & \multicolumn{2}{l}{ Representative transcripts (no.) } & Transcriptome coverage (bp) \\
\cline { 3 - 5 } Species & Assembled transcripts (no.) & Single isoform & Multiple isoforms & Total & Trans \\
\hline P. axillaris & 74,573 & 18,487 & 14,507 & 32,994 & $53,135,953$ \\
P. exserta & 54,913 & 19,517 & 10,708 & 30,225 & $47,589,955$ \\
P. integrifolia & 104,739 & 13,009 & 20,531 & 33,540 & $49,087,745$ \\
\hline
\end{tabular}




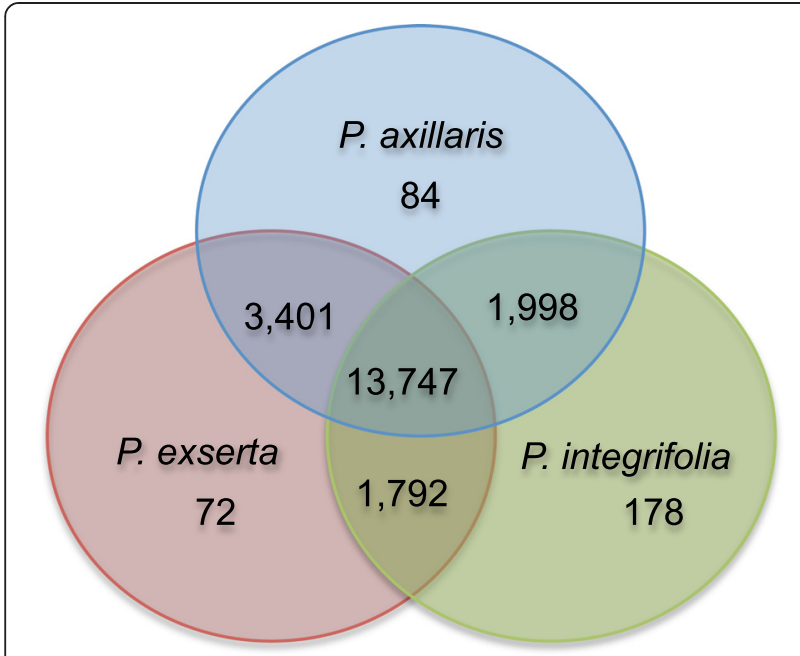

Fig. 2 OrthoMCL identified orthologous gene clusters in the three Petunia species. A total of 21,272 orthologous clusters were identified among all comparisons. Unique and species specific OrthoMCL clusters are shown

among the three species, indicating the uniformity of the transcriptome assemblies and their subsequent annotation (Fig. 3).

\section{SNP detection and characterization}

In the absence of whole genome sequences, comparative transcriptome analysis has proven utility for developing SNP markers [30, 31]. To facilitate genetic analysis within the genus, the three Petunia spp. transcriptomes were utilized for SNP discovery using the Genome Analysis Toolkit (GATK; see Additional file 1: Figure S3) [51]. When utilizing RNA-seq data for SNP discovery, removal of duplicate reads increases SNP detection sensitivity and specificity [52], thus this strategy was adopted resulting in utilization of $62.7 \%$ of $P$. axillaris, $51.3 \%$ of $P$. exserta and $60.8 \%$ of $P$. integrifolia reads that uniquely mapped to the $P$. axillaris transcriptome (data not shown). Depth of sequence coverage increases the reliability of SNP detection [52] and utilization of a minimum threshold for read coverage depth of 10 resulted in mapping of $93.7 \%, 81.2 \%$ and $73.3 \%$ of the reads for $P$. axillaris, $P$. exserta, and $P$. integrifolia, respectively (Table 3).

Overall, there were 814,408 SNPs between the three species. Of these, 105,645 were located in $5^{\prime}$ untranslated regions (UTRs), 481,289 were located within the coding sequence (CDS), and 217,178 were located in 3'UTRs. SNP frequency was calculated by dividing the total length of the reference transcriptome by the total number of SNPs. When only considering the length of 5'UTRs, CDS, and 3'UTRs in transcripts (not the length of the genomic regions these transcripts spanned), the SNP frequencies were $1 \mathrm{SNP} / 69 \mathrm{bp}, 1 \mathrm{SNP} / 61 \mathrm{bp}$ and 1 $\mathrm{SNP} / 62 \mathrm{bp}$ in these regions, respectively. The overall $\mathrm{SNP}$ frequency was $1 \mathrm{SNP} / 63 \mathrm{bp}$.

After filtering, among the 32,994 representative $P$. axillaris transcripts (unigenes), we identified SNPs between $P$. axillaris and either $P$. exserta or $P$. integrifolia in 20,606 unigenes. Gene Ontology (GO) annotation revealed that among all unigenes, 22,535 (68.3 \%) contained GO terms, while 16,787 (80.8 \%) of the SNPcontaining unigenes were assigned with one or more GO ID (Additional file 1: Figure S4). In general, the distribution of GO terms was very similar between all unigenes and those containing SNPs. KEGG Pathway analysis was carried out to determine functional categorization of unigenes containing SNPs. A total of

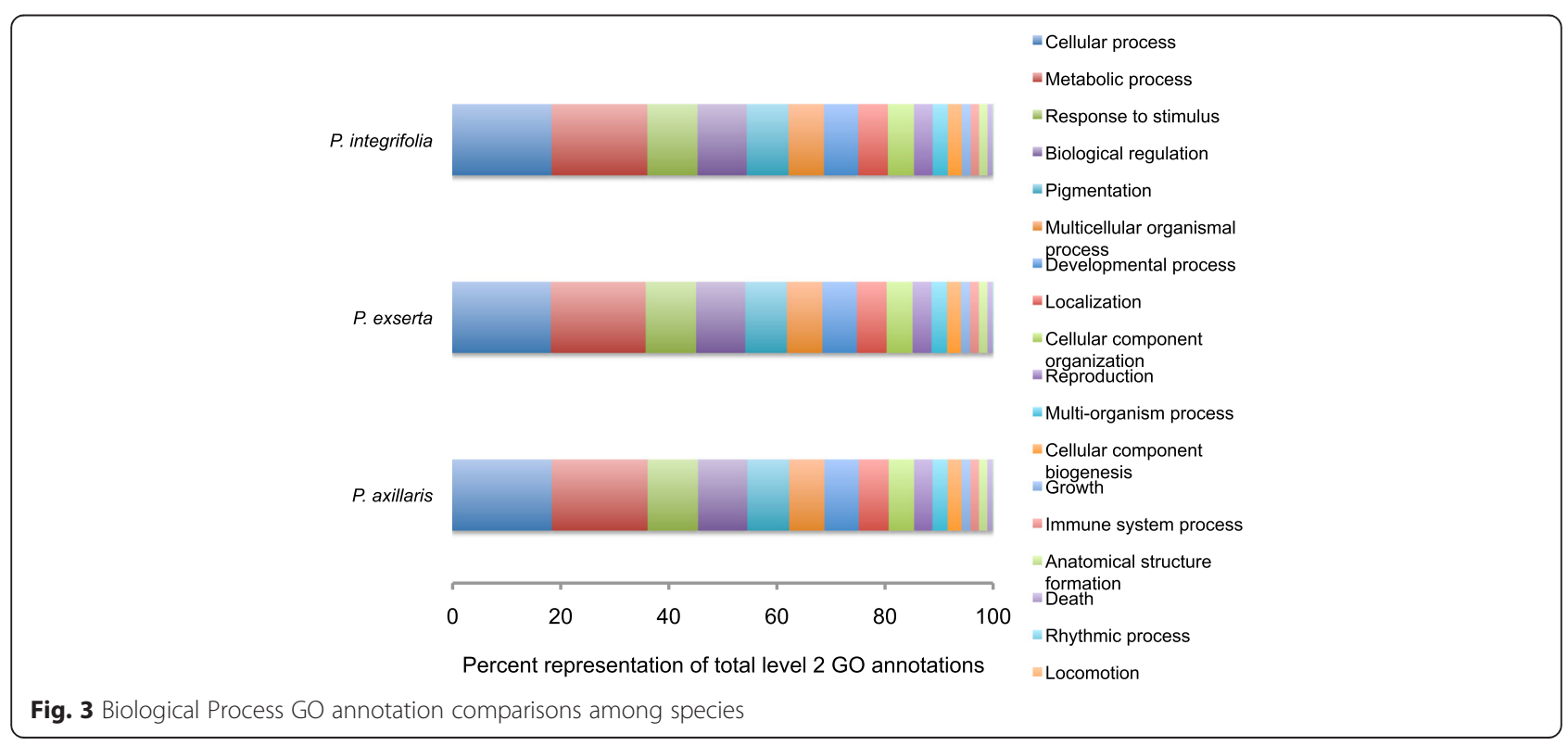


Table 3 Summary of read depth coverage for Petunia axillaris, P. exserta, and P. integrifolia reads mapped to the P. axillaris transcriptome assembly after de-duplication

\begin{tabular}{|c|c|c|c|c|c|c|c|c|c|c|c|}
\hline \multirow[b]{2}{*}{ Sample } & \multirow[b]{2}{*}{ Total reads (millions) } & \multirow[b]{2}{*}{ Mean read depth } & \multicolumn{3}{|c|}{ Granular Quartile Read Depths } & \multicolumn{6}{|c|}{$\begin{array}{l}\% \text { of Reference Transcriptome with Read Depth } \\
\text { Coverage: }\end{array}$} \\
\hline & & & Third (25\%) & Median (50\%) & First (75\%) & $>10$ & $>15$ & $>20$ & $>30$ & $>40$ & $>50$ \\
\hline P. axillaris & 13.35 & 251 & 264 & 93 & 30 & 93.7 & 88.6 & 83.4 & 74.6 & 68.2 & 63.3 \\
\hline P. exserta & 11.00 & 207 & 214 & 70 & 16 & 81.2 & 75.8 & 71.5 & 64.9 & 60.0 & 56.1 \\
\hline P. integrifolia & 7.65 & 144 & 160 & 49 & 9 & 73.3 & 67.9 & 63.9 & 57.8 & 53.2 & 49.4 \\
\hline Total & 32.00 & 355 & & & & & & & & & \\
\hline
\end{tabular}

5558 (27.8\% of total) unigenes containing SNPs were annotated by 2910 KO (KEGG Orthology) identifiers by using only references appropriate for plant species (Table 4). These unigenes were assigned to 322 KEGG pathways. Overall, the SNP frequency for genes involved in metabolism and organismal systems super pathways was lower than those involved in genetic information processing and environmental information processing.

In total, 89,007 SNP positions were detected among $P$. axillaris, $P$. exserta and $P$. integrifolia. Of these, 8868 (10.0\%) were polymorphic across all three species combinations. This calculation was based on positions for which at least one species, $P$. integrifolia or $P$. exserta, had sufficient depth of coverage (10X). After removing low depth of coverage SNPs, 73,193 SNPs remained between $P$. axillaris and $P$. integrifolia, 25,847 SNPs remained between $P$. axillaris and $P$. exserta, and 79,438 SNPs remained between $P$. exserta and $P$. integrifolia. (Fig. 4a).

SNPs were further classified based on their location; CDS or UTR and their zygosity. Altogether, only 10 homozygous SNPs were polymorphic across all three species combinations (Fig. 4b). Between P. axillaris and P. exserta, 24,528 SNPs were homozygous, which comprised $94.9 \%$ of all SNPs. There were 13,512 homozygous SNPs, $17 \%$ of the total SNP positions, between $P$. exserta and $P$. integrifolia. Between $P$. integrifolia and $P$. axillaris, there were only 4539 homozygous SNPs, which constituted only $6.2 \%$ of all polymorphisms, indicating that the self-incompatible $P$. integrifolia is highly heterozygous. Of all SNP loci, 60,701 were located within the CDS, of which 60,561 were biallelic; 9537 were located within 5囚UTRs, of which 9512 were biallelic; 17,983 were located within 3囚UTRs, of which 17,923 were biallelic. There is a small group of 786 SNPs whose location could not be determined.

As with the unfiltered SNPs, SNP frequency was calculated by dividing the total length of the reference transcriptome (Table 2) by the total number of SNPs (Table 5). Overall, the SNP frequency was $1 / 597 \mathrm{bp}$. The SNP frequency was $1 / 2056 \mathrm{bp}$ between $P$. axillaris and $P$. exserta, $1 / 726$ bp between $P$. axillaris and $P$. integrifolia, and $1 / 669$ bp between $P$. integrifolia and $P$. exserta.
All SNPs among the three species were distributed across 20,606 unigenes (62.5\% of the total unigenes), corresponding to $\sim 76 \%(40,556,099 / 53,135,953 \mathrm{bp})$ of the entire unigene length. When only considering the unigenes containing SNPs, the overall SNP frequency was $1 \mathrm{SNP} / 456 \mathrm{bp}(89,007 \mathrm{SNPs} / 40,556,099 \mathrm{bp})$ and the highest SNP frequency was $1 / 89 \mathrm{bp}$. Among the SNPcontaining unigenes, only 1944 (9.4\%) had 10 or more SNPs (Table 5). This suggests that the SNPs were broadly distributed across the transcriptome, which might facilitate SNP marker selection for genome wide association (GWAS) studies.

SNPs between $P$. axillaris and $P$. exserta were distributed across 12,060 unigenes (Table 5). There were only 110 unigenes with 10 or more SNPs. The maximum SNP frequency per unigene was $1 \mathrm{SNP} / 111 \mathrm{bp}$. Between $P$. axillaris and $P$. integrifolia, SNPs were identified in 17,949 unigenes. There were 1466 unigenes with 10 or more SNPs. The maximum SNP frequency per unigene was 1 SNP/96 bp. There were 18,032 unigenes with SNPs between $P$. integrifolia and $P$. exserta, and 1722 unigenes with more than 10 SNPs. The maximum SNP frequency per unigene was $1 / 89 \mathrm{bp}$.

Due to the stringent nature of the SNP discovery parameters employed (i.e., filtering out three SNPs occurring within $100 \mathrm{bp}$ of each other and filtering out SNPs within the first and last of $30 \mathrm{bp}$ of a transcript, etc.), the above SNP frequencies calculated after the filtering steps are likely underestimated. Additionally, for organisms without a fully sequenced genome, using a transcriptome de novo assembly followed by variant detection can result in underestimation of expressed variants [52]. Even with the collection of different tissue types in our study, there are still likely to be undetected variants.

The percentage of unigenes with ten or more SNPs was $0.9 \%, 8.2 \%$ and $7.5 \%$ of the entire SNP-containing unigene set between $P$. axillaris and $P$. exserta, $P$. axillaris and $P$. integrifolia, and $P$. integrifolia and $P$. exserta, respectively (Fig. 5). On average, there were $2.7 \mathrm{SNPs} /$ transcript, with $2.2,0.78$ and $2.4 \mathrm{SNPs} /$ transcript between $P$. axillaris and $P$. integrifolia, $P$. axillaris and $P$. exserta, and $P$. integrifolia and $P$. exserta, respectively. When evaluating the transcripts with more than ten 
Table 4 KAAS (KEGG Automatic Annotation Server) analysis of super pathways involving SNP-containing transcripts

\begin{tabular}{|c|c|c|c|}
\hline Super pathways $^{a}$ & Annotation entries $^{\mathrm{b}}$ & Number of genes & Number of SNPs \\
\hline \multicolumn{4}{|l|}{ Metabolism } \\
\hline Amino acid metabolism & 422 & 322 & 1530 \\
\hline Biosynthesis of other secondary metabolites & 157 & 117 & 449 \\
\hline Carbohydrate metabolism & 702 & 449 & 2451 \\
\hline Energy metabolism & 287 & 265 & 1136 \\
\hline Glycan biosynthesis and metabolism & 129 & 95 & 470 \\
\hline Lipid metabolism & 340 & 242 & 285 \\
\hline Metabolism of cofactors and vitamins & 185 & 185 & 748 \\
\hline Metabolism of other amino acids & 135 & 122 & 547 \\
\hline Metabolism of terpenoids and polyketides & 135 & 134 & 630 \\
\hline Nucleotide metabolism & 193 & 139 & 719 \\
\hline Xenobiotics biodegradation and metabolism & 79 & 44 & 199 \\
\hline \multicolumn{4}{|l|}{ Genetic Information Processing } \\
\hline Folding, sorting and degradation & 455 & 418 & 2289 \\
\hline Replication and repair & 240 & 139 & 914 \\
\hline Transcription & 212 & 212 & 1289 \\
\hline Translation & 492 & 458 & 2432 \\
\hline \multicolumn{4}{|l|}{ Environmental Information Processing } \\
\hline Membrane transport & 23 & 23 & 189 \\
\hline Signal transduction & 970 & 504 & 2719 \\
\hline Signaling molecules and interaction & 3 & 3 & 35 \\
\hline \multicolumn{4}{|l|}{ Cellular Processes } \\
\hline Cellular community & 118 & 63 & 373 \\
\hline Cell growth and death & 351 & 204 & 1202 \\
\hline Cell motility & 60 & 60 & 339 \\
\hline Transport and catabolism & 288 & 266 & 1535 \\
\hline \multicolumn{4}{|l|}{ Organismal System } \\
\hline Development & 72 & 36 & 135 \\
\hline Immune system & 324 & 158 & 811 \\
\hline Environmental adaptation & 183 & 179 & 971 \\
\hline
\end{tabular}

${ }^{\mathrm{a}}$ Only pathways relevant to plants are reported in the table

${ }^{\mathrm{b}}$ Annotation entry is the unique record of the transcript (gene) name and gene Ko number; multiple genes may have the same Ko number

SNPs for GO annotation, the gene percentage in each category was in proportion with the overall transcriptome GO annotation (Additional file 1: Figure S5), indicating no particular set of genes were enriched regarding SNP frequency.

To gain additional insight into base substitutions occurring at each polymorphic site, the transition to transversion ratios (Ts/Tv) were determined (Table 6). Overall, the transition to transversion ratio (Ts/Tv) was 1.7:1. Across all species comparisons, the Ts/Tv was consistently highest for SNPs within CDS, followed by SNPs located in 3区UTRs, while the Ts/Tv was always lowest for SNPs located in 5区UTR. The overall Ts/Tv was relatively stable between species, with a range of 1.6 to 1.8
(Table 6). The Ts/Tv ratio was used as a critical metric for assessing the specificity of new SNP calls in human genome research [53], and might be a useful parameter for subsequent Petunia SNP discovery. Normally, assuming that mutations are completely random, the Ts/Tv would be 0.5 . Our Ts/Tv data indicate that each type of transitional change is produced more than three times as often as each type of transversion. Transitions occurring more frequently than transversions in transcriptome-derived SNPs have also been reported in other plant species, including Chinese pine [54] and melon [55]. The Ts/Tv bias could be the result of a high degree of methyl $\mathrm{C}$ to $\mathrm{U}$ in genomes [56, 57]. In plants, Ts/Tv can vary across species. For example, in the exome assembly of four Neotropical 
A

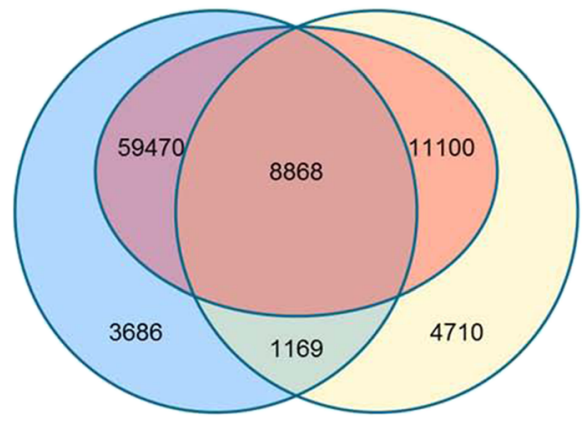

B

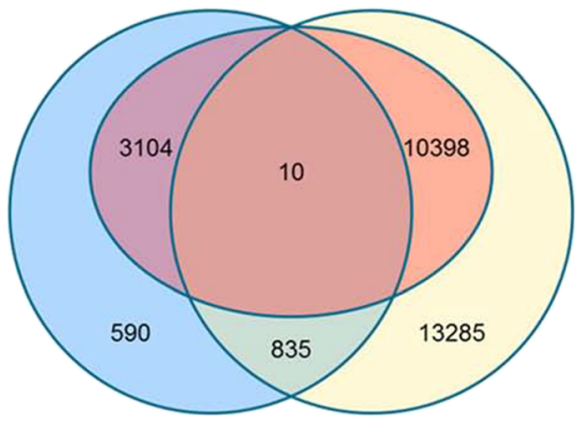

- P. integrifolia $\times$ P. axillaris

- P. axillaris $x$ P. exserta

- P. integrifolia $x$ P. exserta

Fig. 4 Summary of SNP loci between three Petunia spp. a Total number of SNP loci between P. axillaris, $P$. exserta and $P$. integrifolia; $\mathbf{b}$ number of homozygous SNP loci between P. axillaris, P. exserta and P. integrifolia. The intersecting portions of the Venn diagram illustrate the number of common loci between the comparisons

tree species, $\mathrm{Ts} / \mathrm{Tv}$ varied between 1.5 and 1.7 [58]. In the transcriptome from Norway spruce, the Ts/Tv was around 1.2 to 1.5 depending on the sequencing quality cut-offs [59]. It has been suggested that a higher $\mathrm{C} \leftrightarrow \mathrm{T}$ transition can be accompanied by a higher number of Ts. The same situation was observed in our study. For instance, SNPs between $P$. integrifolia and $P$. axillaris, and between $P$. exserta and $P$. integrifolia, had a higher rate of $\mathrm{C} / \mathrm{T}$ mutations than was observed between $P$. axillaris and $P$. exserta, which was accompanied by a slightly higher Ts/Tv.

Minor allele read count frequency was used to calculate the frequency of short reads aligned to the least common allele in all three genotypes. Using a small number of genotypes for SNP discovery, this measure should provide better support for SNP confidence than using minor allele frequency (MAF), where the least prevalent allele frequency is calculated based on the genotype of each individual in a given population. For example, if there are only a few individuals in the panel, the smallest minor allele frequency will be $1 / 2 n$ ( $n$ is the number of individuals), in our case, the minimum MAF would be $16.7 \%$. However, the average depth of coverage was above 100, and for RNA-seq data, the preferentially expressed genes will have a much higher read depth. Thus, even employing a depth cut-off value of 10 , a heterozygous genotype call could have one allele with a much higher read count than the other allele, which might actually be caused by sequencing error or misalignment. This can be detected using the minor allele read count frequency. SNPs with minor allele reads count frequency ranging from 0.3 to 0.5 accounted for $61 \%$ of the total SNPs (Fig. 6).

\section{SNP validation}

A total of 55 primer pairs were selected for PCR amplification. Of these, 50 amplified single DNA fragments, containing a total of 51 SNP loci, matching the expected sizes in $P$. axillaris and were selected for further sequencing (Additional file 1: Table S1). The failed/unclear amplification might be from primers located across intron-exon boundaries, or from the amplification being across a large intron or in paralogous genes. Three of the loci were monomorphic, five yielded poor sequence

Table 5 SNP frequency, the number of unigenes which contain SNPs, and percentage of the total unigene length for all three species combinations

\begin{tabular}{|c|c|c|c|c|c|c|}
\hline Species Comparison & SNP frequency & $\begin{array}{l}\text { SNP-containing } \\
\text { unigenes (no.) }\end{array}$ & $\begin{array}{l}\text { Total unigene length } \\
\text { containing SNPs (\%) }\end{array}$ & $\begin{array}{l}\text { Unigenes with } \\
\geq 10 \text { SNPs (no.) }\end{array}$ & $\begin{array}{l}\text { SNP-containing } \\
\text { unigenes with } \geq 10 \\
\text { SNPs (\%) }\end{array}$ & $\begin{array}{l}\text { Maximum SNP } \\
\text { frequency per } \\
\text { unigene }\end{array}$ \\
\hline Overall & 1/597 bp & 20606 & 76 & 1944 & 9.42 & $1 / 89 \mathrm{bp}$ \\
\hline $\begin{array}{l}\text { P. axillaris and } \\
\text { P. exserta }\end{array}$ & $1 / 2056 \mathrm{bp}$ & 12060 & 49 & 110 & 0.91 & $1 / 111 \mathrm{bp}$ \\
\hline $\begin{array}{l}\text { P. axillaris and } \\
\text { P. integrifolia }\end{array}$ & $1 / 726 \mathrm{bp}$ & 17949 & 70 & 1466 & 8.17 & $1 / 96 \mathrm{bp}$ \\
\hline P. exserta and P. integrifolia & $1 / 669 \mathrm{bp}$ & 18032 & 70 & 1722 & 9.55 & $1 / 89$ bp \\
\hline
\end{tabular}




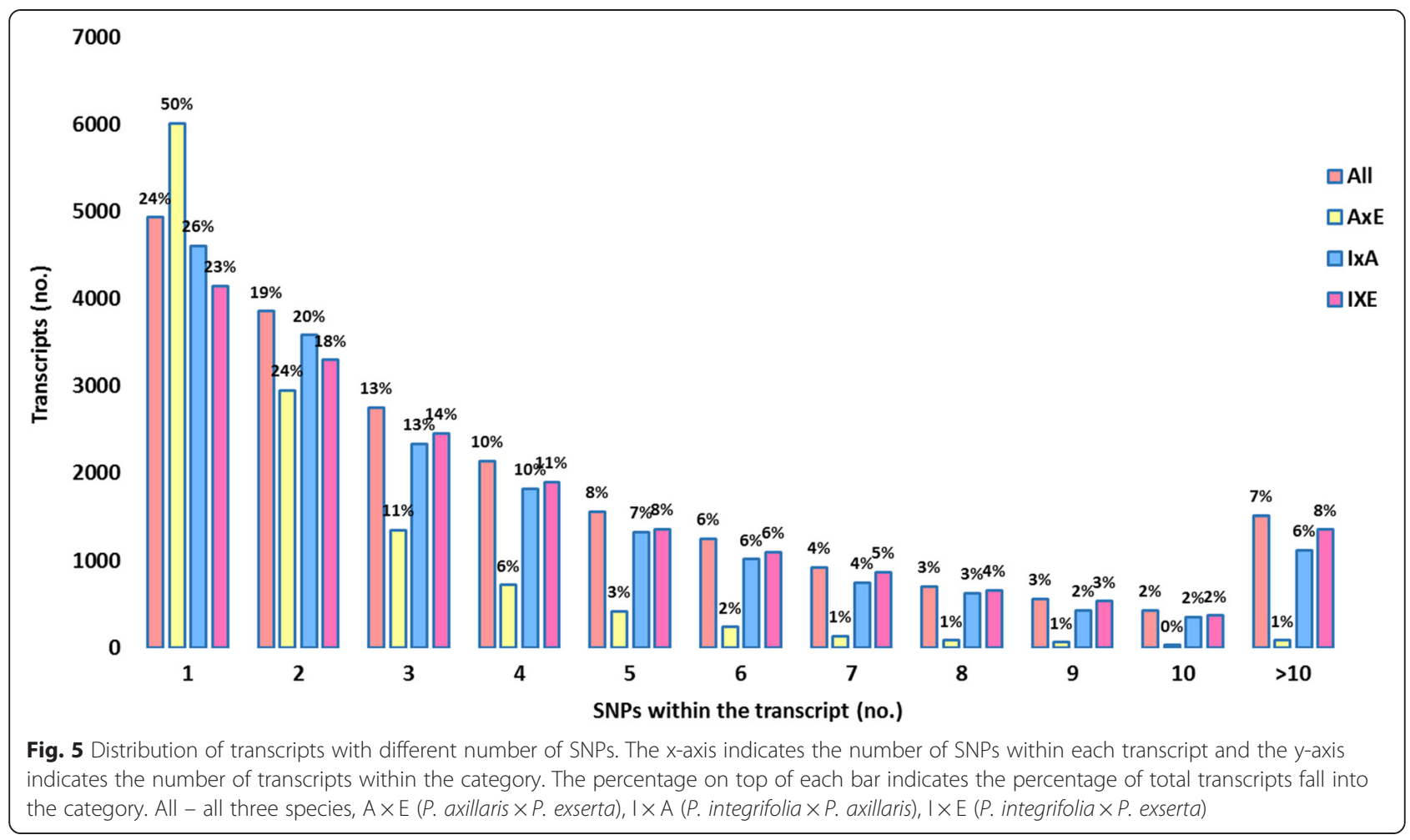

Table 6 SNP distribution and corresponding transition to transversion ratio (Ts/Tv) in coding regions (CDS), 5'UTRs and $3^{\prime} \mathrm{UTRS}$

\begin{tabular}{|c|c|c|c|c|c|c|c|c|c|}
\hline & Number & $\%$ & $\begin{array}{l}\text { A/G } \\
\text { Transition }\end{array}$ & $\begin{array}{l}\mathrm{C} / \mathrm{T} \\
\text { Transition }\end{array}$ & $\begin{array}{l}\text { T/G } \\
\text { Transversion }\end{array}$ & $\begin{array}{l}\text { A/C } \\
\text { Transversion }\end{array}$ & $\begin{array}{l}\mathrm{A} / \mathrm{T} \\
\text { Transversion }\end{array}$ & $\begin{array}{l}\mathrm{C} / \mathrm{G} \\
\text { Transversion }\end{array}$ & $\mathrm{Ts} / \mathrm{TV}$ \\
\hline All SNPs (biallelic) & 88,730 & $99.7 \%^{\mathrm{a}}$ & $31.5 \%$ & $32.0 \%$ & $8.4 \%$ & $8.5 \%$ & $12.1 \%$ & $7.6 \%$ & 1.7 \\
\hline SNPs in CDs (biallelic) & 60,511 & & $32.4 \%$ & $33.2 \%$ & $7.9 \%$ & $8.1 \%$ & $11.3 \%$ & $7.0 \%$ & 1.9 \\
\hline SNPs in 5'UTRs (biallelic) & 9512 & & $28.1 \%$ & $29.3 \%$ & $9.5 \%$ & $9.6 \%$ & $14.1 \%$ & $9.3 \%$ & 1.4 \\
\hline SNPs in 3'UTRs (biallelic) & 17,923 & & $30.2 \%$ & $29.4 \%$ & $9.5 \%$ & $8.9 \%$ & $13.6 \%$ & $8.5 \%$ & 1.5 \\
\hline $\begin{array}{l}\text { P. axillaris and } \\
\text { P. exserta (biallelic) }\end{array}$ & 25,650 & $99.2 \%$ & $30.5 \%$ & $31.2 \%$ & $8.9 \%$ & $9.1 \%$ & $12.2 \%$ & $8.0 \%$ & 1.6 \\
\hline SNPs in CDs (biallelic) & 14,686 & $99.1 \%$ & $31.7 \%$ & $32.9 \%$ & $8.4 \%$ & $8.8 \%$ & $11.0 \%$ & $7.2 \%$ & 1.8 \\
\hline SNPs in 5'UTRs (biallelic) & 3842 & $99.5 \%$ & $27.6 \%$ & $29.1 \%$ & $10.0 \%$ & $10.2 \%$ & $13.9 \%$ & $9.2 \%$ & 1.3 \\
\hline SNPs in 3'UTRs (biallelic) & 6753 & $99.3 \%$ & $29.7 \%$ & $28.9 \%$ & $9.6 \%$ & $9.0 \%$ & $14.0 \%$ & $8.8 \%$ & 1.4 \\
\hline $\begin{array}{l}\text { P. integrifolia and } \\
\text { P. axillaris (biallelic) }\end{array}$ & 72,926 & $99.6 \%$ & $31.8 \%$ & $32.3 \%$ & $8.2 \%$ & $8.3 \%$ & $12.0 \%$ & $7.5 \%$ & 1.8 \\
\hline SNPs in CDs (biallelic) & 52,413 & $99.7 \%$ & $32.6 \%$ & $33.3 \%$ & $7.8 \%$ & $8.0 \%$ & $11.3 \%$ & $7.0 \%$ & 1.9 \\
\hline SNPs in 5'UTRs (biallelic) & 6765 & $99.7 \%$ & $28.7 \%$ & $29.5 \%$ & $9.0 \%$ & $9.4 \%$ & $14.2 \%$ & $9.3 \%$ & 1.4 \\
\hline SNPs in 3'UTRs (biallelic) & 13,263 & $99.6 \%$ & $30.3 \%$ & $29.6 \%$ & $9.5 \%$ & $8.9 \%$ & $13.5 \%$ & $8.3 \%$ & 1.5 \\
\hline $\begin{array}{l}\text { P. exserta and } P \text {. integrifolia } \\
\text { (biallelic) }\end{array}$ & 79,182 & $99 . \%$ & $31.8 \%$ & $32.3 \%$ & $8.3 \%$ & $8.3 \%$ & $11.9 \%$ & $7.4 \%$ & 1.8 \\
\hline SNPs in CDs (biallelic) & 56,820 & $99.7 \%$ & $32.7 \%$ & $33.3 \%$ & $7.9 \%$ & $8.0 \%$ & $11.2 \%$ & $6.9 \%$ & 1.9 \\
\hline SNPs in 5'UTRs (biallelic) & 7396 & $99.7 \%$ & $28.2 \%$ & $29.8 \%$ & $9.4 \%$ & $9.3 \%$ & $14.0 \%$ & $9.3 \%$ & 1.4 \\
\hline SNPs in $3^{\prime}$ UTRs (biallelic) & 14,451 & $99.6 \%$ & $30.2 \%$ & $29.6 \%$ & $9.5 \%$ & $8.8 \%$ & $13.6 \%$ & $8.3 \%$ & 1.5 \\
\hline
\end{tabular}

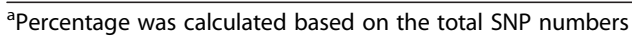




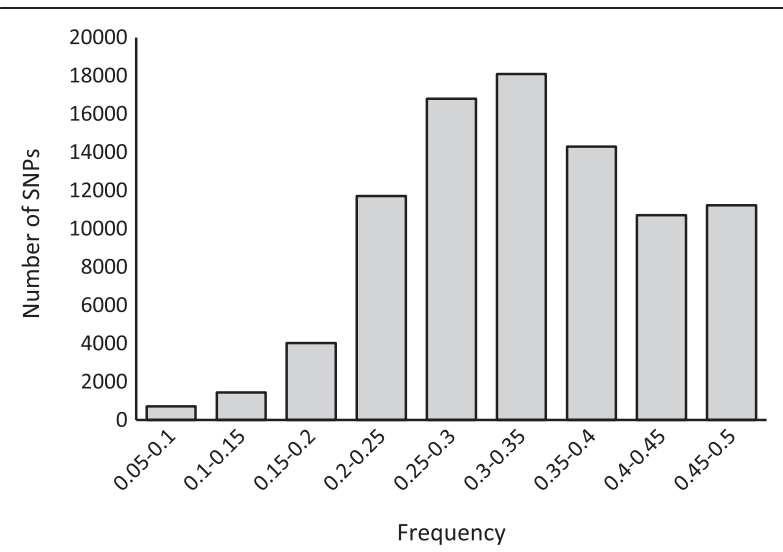

Fig. 6 Minor allele reads count frequency distribution for SNPS among $P$. axillaris, P. exserta, and P. integrifolia

quality, which was associated with either failure of the sequence reaction or ambiguous base calls that were likely due to heterozygosity of the parents that resulted in amplification of distinct alleles of a given locus. The remaining 43 loci were polymorphic between at least two species resulting in an overall SNP validation rate of $84.3 \%$ (43/51). For the three monomorphic SNP loci, the minor allele sequence read count frequencies were $0.07,0.26$ and 0.33 , indicating that low minor allele sequence read count frequency might be one factor that can introduce false positives in SNP identification. The validation rate obtained in this study is comparable with previously reported results for transcriptome derived SNPs. For example, the validation rate for pea (Pisum sativum) transcriptome derived SNPs was $84.5 \%$ by selecting a 1920 SNPs set in a GoldenGate VeraCode assay on a large number of pea accessions [60]. The validation rate for the SNP detected from Douglas-fir (Pseudotsuga menziesii) was $72.5 \%$ [61] while, in potato, the validation rate was $85 \%$ for set of 96 transcriptome derived SNPs [62].

\section{CAPS marker design and validation}

Twenty restriction enzymes were used to computationally predict CAPS markers from 15,701 SNP loci (Additional file 5: Dataset S4). Some SNP loci are predicted to be digested by two different restriction enzymes, with one recognizing the reference allele and the other recognizing the alternative allele, or by different restriction enzymes with overlapping recognition sequences. For example, for the restriction enzymes $A l u \mathrm{I}$ and $S a c \mathrm{I}, S a c \mathrm{I}$ is a 6 bp cutter recognizing/cutting sequences of $\mathrm{GAGCT}^{\vee} \mathrm{C}$ and $A l u \mathrm{I}$ is a 4 bp cutter that recognizes/cuts $\mathrm{AG}^{\vee} \mathrm{CT}$, thus all $A l u \mathrm{I}$ recognition cites are also recognized by SacI. These analyses led to the identification of a total of 17,635 predicted CAPS markers (Supplemental dataset 4). Fourteen putative CAPS markers were randomly selected for validation.
All primer pairs amplified products of the expected size, and 11 were polymorphic. All 14 amplicons for P. axillaris and $P$. exserta had the expected genotype, while four amplicons from $P$. integrifolia were homozygous while the genotype prediction was heterozygous. However, the CAPS markers generated from this study have some limitations. First, the CAPS marker design pipeline only considered possible restriction enzyme recognition differences between species, the number/size of the fragments generated from digestion was not taken into consideration. Thus, there might be multiple small fragments yielded from the digestion, or the digested fragments between genotypes may only vary by a few base pairs. Either situation could not be scored successfully on agarose gel genotyping systems. Second, there could be sequence differences between the $P$. axillaris reference genome and the actual parental genotype used for this study (a different $P$. axillaris accession), and some restriction enzyme recognition sites predicted in the pipeline might not exist when screening our samples.

\section{Simple sequence repeat (SSR) marker development}

Of the 32,994 P. axillaris unigenes, 3027 ( 9 \%) contain predicted SSRs, and $375(\sim 12 \%)$ of these possess more than one SSR (Table 7). The SSR frequency in the P. axillaris transcriptome is approximately $1 \mathrm{SSR}$ per $15.2 \mathrm{~kb}$, which is one-tenth of the SNP frequency between $P$. axillaris, $P$. integrifolia and $P$. exserta. The SSR frequency in $P$. axillaris is lower than the frequency in cassava (Manihot esculenta; $7.0 \mathrm{~kb}$ ) [63], coffee (Coffea spp.; $7.7 \mathrm{~kb})$ [64] and peanut (Arachis hypogaea) (5 kb) [65]. Among the $P$. axillaris SSRs, $\sim 42 \%$ are di-nucleotide repeats, of which AG/CT is most prevalent (43\%) and $~ 53 \%$ are trinucleotide repeats with AAG/CTT repeats present at the highest frequency ( 17.5\%; Table 8$)$.

A total of 2949 primer pairs were developed from 3027 unigenes (Additional file 6: Dataset S5). The designed SSR primer pairs included 1,481 di-, 1,867 tri-, 88

Table 7 Summary of SSR markers in the P. axillaris

transcriptome

\begin{tabular}{ll}
\hline Total number of sequences examined & 32,994 \\
Total size of examined sequences (bp) & $53,135,953$ \\
Total number of identified SSRs & 3499 \\
Number of SSR containing sequences & 3027 \\
Number of sequences containing more than 1 SSR & 375 \\
Number of SSRs present in compound formation & 206 \\
Di-nucleotide & 1481 \\
Tri-nucleotide & 1867 \\
Tetra-nucleotide & 88 \\
Penta-nucleotide & 16 \\
Hexa-nucleotide & 47 \\
\hline
\end{tabular}


Table 8 Summary of SSR repeat motif types and their corresponding repeat unit numbers for di- and tri- nucleotide repeats

\begin{tabular}{|c|c|c|c|c|c|c|c|c|c|c|c|c|c|c|c|c|c|}
\hline Repeats & 5 & 6 & 7 & 8 & 9 & 10 & 11 & 12 & 13 & 14 & 15 & 16 & 17 & 18 & 19 & 20 & Total \\
\hline $\mathrm{AC} / \mathrm{GT}$ & - & 203 & 132 & 76 & 56 & 38 & 15 & 15 & 6 & 7 & 7 & 4 & 2 & 2 & 1 & & 564 \\
\hline $\mathrm{AG} / \mathrm{CT}$ & - & 209 & 130 & 81 & 40 & 34 & 25 & 17 & 17 & 14 & 13 & 10 & 4 & 5 & 9 & 3 & 611 \\
\hline AT/TA & - & 122 & 64 & 29 & 19 & 10 & 9 & 8 & 7 & 3 & 2 & 1 & 1 & & & 1 & 276 \\
\hline $\mathrm{AAC/GTT}$ & 186 & 72 & 23 & 17 & 8 & 5 & 4 & 4 & 4 & 1 & 1 & & & 1 & & 2 & 328 \\
\hline $\mathrm{AAG} / \mathrm{CT}$ & 205 & 68 & 35 & 11 & 6 & 6 & 7 & 4 & 3 & & 2 & 1 & 6 & 3 & 1 & 3 & 361 \\
\hline AAT/ATT & 107 & 47 & 24 & 6 & 3 & 3 & 3 & & 1 & 1 & & & & & 2 & 2 & 199 \\
\hline ACC/GGT & 159 & 56 & 21 & 4 & 1 & 1 & & & & & & & & & & & 242 \\
\hline ACG/CGT & 7 & 2 & 1 & & 1 & & & & & & & & & & & & 11 \\
\hline $\mathrm{ACT} / \mathrm{AGT}$ & 77 & 24 & 7 & 11 & 4 & & & 1 & 1 & & & & & & 1 & 1 & 127 \\
\hline AGC/CTG & 104 & 33 & 15 & 9 & 7 & 1 & 2 & & & & & & 1 & & & & 172 \\
\hline AGG/CCT & 72 & 17 & 7 & 7 & 3 & 1 & 1 & & & & & & & & & & 108 \\
\hline ATC/ATG & 182 & 68 & 17 & 8 & 10 & 3 & 1 & 1 & & 1 & & & & & & & 291 \\
\hline $\mathrm{CCG} / \mathrm{CGG}$ & 19 & 2 & 1 & 1 & & & & & & & & & & & & & 23 \\
\hline
\end{tabular}

tetra-, 16 penta-, and 47 hexa- nucleotide repeats (Table 7). Forty-eight SSR markers were randomly selected for their utility in petunia (Additional file 7: Dataset S6). Of these, 36 (75\%) were successfully amplified and displayed polymorphisms between at least two of the three species examined. For example, 24 SSRs were polymorphic between $P$. integrifolia and $P$. axillaris, 28 were polymorphic between $P$. axillaris and $P$. exserta, and 24 were polymorphic between $P$. integrifolia and $P$. exserta. This success rate was higher than for our previous results developing SSR markers from $P$. axillaris ESTs [66].

Representation and in silico expression analysis of Petunia plastochron-related transcripts

Several genes from Arabidopsis are known to influence plastochron (Table 9). Local tBLASTN searches of the Petunia spp. transcriptomes using 14 Arabidopsis plastochron-related proteins as the query sequences revealed the presence of highly homologous unigenes within each transcriptome (Table 9). The majority (34 out of 46) of the recovered transcripts are predicted to encode full-length proteins despite the fact that many plastochron-related genes, including ERECTA, ERECTA LIKE-1, SERRATE and SLOW MOTION, yield transcripts close to $3 \mathrm{~kb}$ or greater. Based on predicted amino sequence alignments, an additional five unigenes appear to be truncated by approximately 60 nucleotides (20 amino acids) or less at their N-termini (Table 9). This targeted analysis supports the transcriptome wide assessment of quality using CEGMA analysis (Fig. 1c) and indicates an overall high quality assembly of each transcriptome. Several of the previously characterized plastochron-related genes are preferentially expressed within the shoot apical meristem or developing leaf primordia [16, 18, 20]. Congruent with these previous findings, among the plastochron-related homologs identified in petunia, those related to AMP1, ERECTA, PIN1, TEL1, KLUH, SERRATE and SPL15 display enriched expression within shoot apices compared to the additional tissues examined (Table 9). These data suggest conservation of both gene content and expression pattern of plastochron-related genes between petunia and Arabidopsis.

\section{Mapping plastochron-related homologous transcripts in an interspecific Petunia population}

We identified Petunia transcripts homologous to numerous plastochron-related genes from Arabidopsis (Table 9). With the previously identified SNPs, 13 of these transcripts were converted to CAPS markers and used for linkage mapping (Additional file 1: Table S2). Together with the previously published SSR and CAPS markers [11], we constructed a linkage map with a total length of 289 cM consisting of 90 markers. Of those, eight were CAPS markers developed from plastochron-related transcripts located on five chromosomes (1, 2, 3, 6, and 7). The average linkage group length was $41.3 \mathrm{cM}$ with a range from $32.7 \mathrm{cM}$ (Chr7) to $58.7 \mathrm{cM}$ (Chr5). The average marker density was $3.2 \mathrm{cM}$.

Similar to our previous study [11], three QTL for development rate located on chromosomes 1, 2 and 5 were detected. Together they explained $37 \%$ of the variation for development rate (Fig. 7; Table 10). However, the QTL location on chromosome 1 shifted away from a CAPS marker developed from the isopentenyl transferase gene $\mathrm{SHO}$, a gene originally identified in an activation-tagged line exhibiting increased lateral shoot production [67]. We found that although markers developed from plastochron-related genes were located on 
Table 9 Petunia homologs of plastochron-related genes from Arabidopsis

\begin{tabular}{|c|c|c|c|c|c|c|}
\hline \multirow[b]{2}{*}{ Unigene identifier } & \multirow[b]{2}{*}{ Annotation } & \multirow[b]{2}{*}{ Coverage $^{a}$} & \multicolumn{4}{|c|}{ Tissue type } \\
\hline & & & Flower & Shoot apices & Seedlings & Trichomes \\
\hline Paxi_locus_37_Transcript_7/9_Length_3129 & $\mathrm{AMP1} 1^{\mathrm{C}}$ & ++ & $16.7^{e}$ & 41.8 & 30.1 & 13.6 \\
\hline Pexs_locus_18730_Transcript_7/8_Length_2607 & AMP1 & ++ & 23.4 & 46.4 & 14.2 & 32.6 \\
\hline Pint_locus_21727_Transcript_1/5_Length_1316 & $A M P 1^{d}$ & - & 6.2 & 21.9 & 8.9 & 3.8 \\
\hline Pint_locus_22203_Transcript_1/2_Length_1109 & AMP1 & - & 5.1 & 13.8 & 8.9 & 3.4 \\
\hline Paxi_locus_7774_Transcript_1/1_Length_3771 & SLOMO & ++ & 26.7 & 22.2 & 23.7 & 20.3 \\
\hline Pexs_locus_3846_Transcript_1/2_Length_3848 & SLOMO & ++ & 29.7 & 24.2 & 19.9 & 25.6 \\
\hline Pint_locus_6038_Transcript_3/3_Length_3716 & SLOMO & ++ & 28.1 & 31.6 & 26 & 25.4 \\
\hline Paxi_locus_28305_Transcript_3/3_Length_4072 & ER & ++ & 33.9 & 81.5 & 22.2 & 29.4 \\
\hline Pexs_locus_27971_Transcript_3/3_Length_4072 & ER & ++ & 22.8 & 55.5 & 8.0 & 66.5 \\
\hline Pint_locus_23938_Transcript_2/6_Length_3741 & ER & ++ & 21.7 & 113 & 35.3 & 37.3 \\
\hline Paxi_locus_17737_Transcript_3/3_Length_3550 & ERL1 & ++ & 13.1 & 33.1 & 4.3 & 62.8 \\
\hline Pexs_locus_28063_Transcript_1/2_Length_3819 & ERL1 & - & 7.8 & 16 & 1.3 & 54.8 \\
\hline Pint_locus_64973_Transcript_1/10_Length_3648 & ERL1 & ++ & 5.4 & 33 & 6.9 & 118.8 \\
\hline Paxi_locus_17054_Transcript_3/3_Length_2365 & PIN1 & ++ & 26.3 & 42.8 & 17.3 & 14.1 \\
\hline Pexs_locus_18753_Transcript_1/1_Length_2550 & PIN1 & ++ & 16.7 & 40.5 & 11.6 & 27.3 \\
\hline Pint_locus_7020_Transcript_2/7_Length_2155 & PIN1 & + & 29 & 51.5 & 35.9 & 12 \\
\hline Paxi_locus_4307_Transcript_1/3_Length_2763 & PIN3 & ++ & 28.5 & 13.8 & 24.6 & 23.3 \\
\hline Pexs_locus_32081_Transcript_2/3_Length_2800 & PIN3 & ++ & 23.0 & 20.8 & 17.1 & 19.0 \\
\hline Pint_locus_15123_Transcript_1/7_Length_3107 & PIN3 & ++ & 56.6 & 35.6 & 146.5 & 27.8 \\
\hline Paxi_locus_2898_Transcript_1/1_Length_2484 & TEL1 & ++ & 0.6 & 11 & 0.5 & 2.5 \\
\hline Pexs_locus_31850_Transcript_1/1_Length_2212 & TEL1 & + & 0 & 9.7 & 0.8 & 10.8 \\
\hline Pint_locus_19236_Transcript_1/3_Length_2722 & TEL1 & ++ & 0 & 9.3 & 0.9 & 2.0 \\
\hline Paxi_locus_11865_Transcript_1/1_Length_1775 & $\mathrm{KLU}$ & ++ & 5.7 & 46.1 & 11.2 & 6.6 \\
\hline Pexs_locus_32423_Transcript_1/1_Length_2010 & $\mathrm{KLU}$ & + & 3.2 & 29.2 & 5.4 & 17.8 \\
\hline Pint_locus_55018_Transcript_1/1_Length_1785 & $\mathrm{KLU}$ & + & 4.0 & 52.5 & 13.1 & 8.6 \\
\hline Paxi_locus_2128_Transcript_1/2_Length_3063 & SER ${ }^{f}$ & ++ & 41.3 & 84.5 & 53.5 & 51.4 \\
\hline Pexs_locus_1424_Transcript_1/1_Length_2854 & SER & ++ & 31.7 & 63.8 & 31.5 & 54.2 \\
\hline Pint_locus_294_Transcript_1/3_Length_2067 & SER & - & 30.7 & 78.5 & 43.9 & 38 \\
\hline Paxi_locus_23917_Transcript_1/1_Length_2956 & SER & - & 1.7 & 9.2 & 2.8 & 2.2 \\
\hline Pexs_locus_14709Transcript_1/1_Length_2964 & SER & ++ & 1.8 & 10 & 1.8 & 7.6 \\
\hline Pint_locus_12396_Transcript_1/3_Length_2121 & SER & - & 2.4 & 19.1 & 4.3 & 5.4 \\
\hline Paxi_locus_1735_Transcript_5/6_Length_4646 & SPL1 & ++ & 77.4 & 37.3 & 43.8 & 44.9 \\
\hline Pexs_locus_1184_Transcript_1/1_Length_3442 & SPL1 & ++ & 17.7 & 21.8 & 8.2 & 18.2 \\
\hline Pint_locus_16_Transcript_3/5_Length_3091 & SPL1 & ++ & 76.5 & 64.3 & 51.4 & 65.8 \\
\hline Paxi_locus_34631_Transcript_2/2_Length_962 & SPL3 & ++ & 14.7 & 8.2 & 1.9 & 5.2 \\
\hline Pexs_locus_31900_Transcript_1/1_Length_1186 & SPL3 & - & 294.9 & 110.5 & 220.0 & 183.8 \\
\hline Pint_locus_74351_Transcript_1/6_Length_1207 & SPL3 & - & 21.1 & 32.1 & 4.5 & 14.9 \\
\hline Paxi_locus_11501_Transcript_1/4_Length_1639 & SPL9 & ++ & 36.5 & 33.1 & 3.9 & 11.9 \\
\hline Pexs_locus_21068_Transcript_1/2_Length_1577 & SPL9 & ++ & 26.4 & 33.2 & 2.4 & 49.7 \\
\hline Pint_locus_21057_Transcript_2/3_Length_1378 & SPL9 & ++ & 22.7 & 42.7 & 3.5 & 9.0 \\
\hline Paxi_locus_30395_Transcript_1/2_Length_1427 & SPL15 & ++ & 3.5 & 34.1 & 1.4 & 3.5 \\
\hline Pexs_locus_31561_Transcript_1/1_Length_1265 & SPL15 & ++ & 3.1 & 20.5 & 1.2 & 19.8 \\
\hline Pint_locus_74440_Transcript_1/2_Length_1316 & SPL15 & ++ & 2.3 & 28.2 & 1.3 & 3.4 \\
\hline
\end{tabular}


Table 9 Petunia homologs of plastochron-related genes from Arabidopsis (Continued)

\begin{tabular}{lllllll}
\hline Pax_locus_6323_Transcript_2/3_Length_3369 & ML5 & ++ & 6.5 & 10.2 & 8.0 & 8.6 \\
Pexs_locus_16730_Transcript_1/2_Length_3261 & ML5 & ++ & 7.1 & 10.7 & 5.7 & 11.2 \\
Pint_locus_1362_Transcript_3/3_Length_3267 & ML5 & ++ & 6.5 & 13.1 & 8.4 & 9.7
\end{tabular}

${ }^{a}$ Coverage refers to whether a unigene encodes a predicted full-length protein $(++)$, is truncated by $\sim 20$ amino acids or less $(+)$, or is truncated by greater than 20 amino acids (-)

${ }^{\mathrm{b}}$ The designation Paxi, Pexs and Pint refer to unigenes derived from P. axillaris, P. exserta and P. integrifolia, respectively

'Gene symbols are as follows: ALTERED MERISTEM PROGRAM 1 (AMP1), SLOW MOTION (SLOMO), ERECTA (ER), ERECTA LIKE 1 (ERL1), PIN FORMED 1 (PIN1), TERMINAL

EAR LIKE 1 (TEL1), KLUH (KLU), SERRATE (SER), SQUAMOSA PROMOTER BINDING PROTEIN-LIKE (SPL), PIN FORMED 3 (PIN3), MEI2-LIKE PROTEIN 5 (ML5)

${ }^{\mathrm{d}}$ Two fragmented unigenes corresponding to $A M P 1$ are present within the $P$. integrifolia transcriptome

${ }^{\mathrm{e}}$ Transcript abundance was determined by RNAseq and data is presented as Fragments Per Kilobase of transcript per Million mapped reads

${ }^{\mathrm{f}}$ Two paralogs with homology to SERRATE are present within each Petunia transcriptome

the same chromosomes as two of the QTL, they did not co-localize with development rate QTL regions. Linkage maps with higher resolution will help better understand the mechanisms for this trait.

\section{Conclusions}

High quality transcriptome assemblies were generated by RNA-seq data from three Petunia spp. and these were mined for molecular markers and plastochronrelated transcripts. We used a SNP discovery pipeline to identify over 80,000 SNPs across three species. As the majority of the SNPs were located in exons and over $80 \%$ of the SNP-containing transcripts can be annotated, these SNPs could possess utility in future gene discovery and breeding applications. Thirteen percent of the identified SNPs were polymorphic among all three species, and can be further used for comparative genomics and population diversity studies. Additionally, over 10,000 SNPs were transformed into CAPS markers and an additional set of SSR markers were developed. Plastochron-related transcripts were identified, converted to CAPS markers, and genetically mapped in a $P$. integrifolia $\times P$. axillaris $F_{2}$ population. Overall, these data provide sequence, expression information and a large marker resource for the Petunia community.

\section{Methods}

\section{Plant material and growth conditions}

Seeds of Petunia axillaris (PI667515) and Petunia exserta (OPGC943) were obtained from the Germplasm Resources Information Network. Seeds of Petunia integrifolia were purchased from Diane's Flower Seeds (Ogdon, UT). Five different tissue types were harvested for RNAseq: 1) whole seedlings, 2) callus, 3) shoot apices, 4) flowers of mixed developmental stages, and 5) trichomes. For whole seedlings, $P$. axillaris, $P$. integrifolia and $P$. exserta seeds were sown on $100 \mathrm{~mm}$ diameter Petri plates containing half-strength Murashige and Skoog (MS) media. Plates were left unsealed to allow air exchange and grown under a $16-\mathrm{h}$ photoperiod provided by fluorescent lamps (ca. $75 \mu \mathrm{mol} \mathrm{m}{ }^{-2} \mathrm{~s}^{-1}$ ) at $22^{\circ} \mathrm{C}$. The date when seeds began to germinate was recorded (generally 2-3 d after sowing) and seedlings where harvested $21 \mathrm{~d}$ after this date. At harvest, media was removed from the roots, and plants were placed in $1.5 \mathrm{ml}$ tubes and immediately frozen with liquid nitrogen.

For callus generation, seeds of each species were sterilized by: 1) soaking in sterilized water for $30 \mathrm{~min}, 2$ ) rinsing with $90 \%$ ethanol, 3) soaking in $50 \%$ bleach for 7 min and 4) rinsing with sterilized water. Twenty seeds per species were sown in a magenta box containing

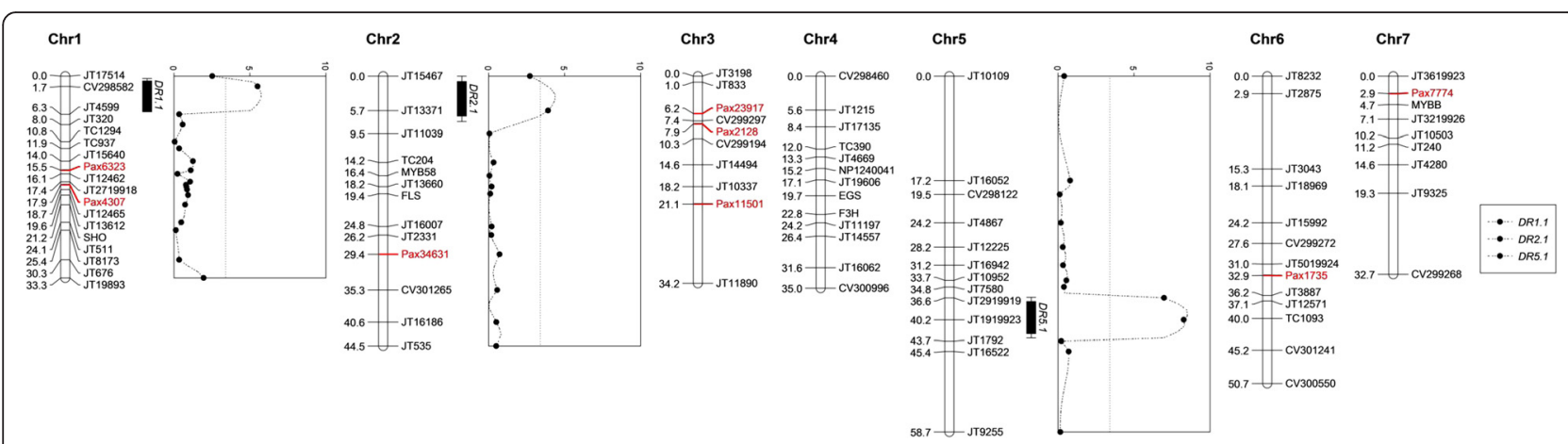

Fig. 7 Genetic linkage map of a P. integrifolia $\times$ P. axillaris $F_{2}$ population. Markers developed from plastochron-related genes are highlighted in red. QTL for development rate (DR) are indicated along with the corresponding LOD score along the linkage group 
Table 10 Summary of QTL for development rate (DRate; in nodes day ${ }^{-1}$ ) in a P. integrifolia $\times$ P. axillaris $F_{2}$ population

\begin{tabular}{lllllcrrr}
\hline Trait & LOD threshold & QTL & LG & Nearest marker & Position (cM) & LOD & $a^{\text {a }}$ & \%VE \\
\hline DRate & 3.4 & DR1.1 & 1 & CV298582 & 1.73 & 5.73 & -0.03 & 11.3 \\
& & DR2.1 & 2 & JT13371 & 4.00 & 4.36 & -0.03 & 8.4 \\
& DR5.1 & 5 & JT1919923 & 39.59 & 8.51 & 0.05 & 17.5 \\
\hline
\end{tabular}

${ }^{a}$ Additive effect of the $P$. integrifolia allele

${ }^{b}$ Percentage of variation explained

$55 \mathrm{ml}$ media [2.22 g MS, $15 \mathrm{~g}$ sucrose and $6 \mathrm{~g}$ agar per liter] and germinated under a $16 \mathrm{~h}$ photoperiod provided by fluorescent lamps (ca. $30 \mu \mathrm{mol} \mathrm{m} \mathrm{m}^{-2} \mathrm{~s}^{-1}$ ) at $22{ }^{\circ} \mathrm{C}$. When seedlings reached a sufficient size, $1 \mathrm{~cm}^{2}$ leaf pieces were excised and placed onto $100 \times 15 \mathrm{~mm}$ plates containing $25 \mathrm{ml}$ callus-induction media [4.44 g MS, $100 \mu \mathrm{g}$ 2,4-D, $20 \mathrm{~g}$ sucrose, and $8 \mathrm{~g}$ agar per liter] (Rao et al., 1973) and incubated in the dark at room temperature. Callus developed after approximately four weeks. Every few weeks until harvest, callus pieces were transferred to new plates containing the same media.

For the collection of shoot apices, flowers and trichomes, seed was sown in 288-cell plug trays in a soilless media and placed under in a greenhouse under intermittent mist at $24{ }^{\circ} \mathrm{C}$. After emergence of the first true leaf, seedlings were removed from mist and placed in a glass-covered greenhouse at $20{ }^{\circ} \mathrm{C}$ under a $16 \mathrm{~h}$ photoperiod under ambient light, supplemented with $60 \mu \mathrm{mol} \mathrm{m} \mathrm{m}^{-2} \mathrm{~s}^{-1}$ provided by high-pressure sodium lamps. Shoot apices were collected when plants had unfolded 6-8 true leaves. Small leaves were removed with forceps to minimize contamination with leaf petiole tissue. The shoot apices from ca. 100 plants of each species were excised, placed in $15 \mathrm{ml}$ conical tubes, and immediately frozen in liquid nitrogen. Flowers were harvested when a full range of reproductive stages of development were present, ranging from flower buds ca. $5 \mathrm{~mm}$ in length through the beginning fruit development. Harvested flowers were placed in paper envelopes, and frozen in liquid nitrogen. In order to harvest trichomes, stems and petioles from flowering Petunia plants were cut into ca. $5 \mathrm{~cm}$ pieces and immediately frozen in liquid nitrogen. The frozen tissue pieces were held using chilled forceps and the trichomes were removed from the stems by gentle scraping with a frozen paint brush and were collected in a mortar containing liquid nitrogen. Trichomes were immediately ground and transferred to $2 \mathrm{ml}$ crew cap tubes for storage at $-80{ }^{\circ} \mathrm{C}$.

\section{RNA extraction}

Total RNA was extracted from each tissue sample using the Trizol $^{\odot}$ reagent (LifeTechnologies) following the manufacturer's instructions. Forty micrograms of RNA was treated for DNA contamination using RNase-free DNase set (Qiagen). DNase-treated RNA was purified using the RNeasy ${ }^{\circ}$ MinElute Cleanup kit (Qiagen). RNA yield and quality were determined using gel electrophoresis, spectroscopy and the Agilent 2100 BioAnalyzer RNA 6000 Pico chip with RIN values $\geq 8.0$ achieved (Agilent Technologies).

\section{RNA-seq library construction, sequencing, and analysis}

A TruSeq RNA Sample Preparation kit (Illumina) was used to construct the cDNA libraries for sequencing. Illumina barcodes allowed pooling of 7-8 libraries per lane. Sequencing was completed on the Illumina HiSeq 2500 next-generation sequencer to $100 \mathrm{nt}$ paired-end at the Michigan State University Research Technology Support Facility (RTSF; http://rtsf.msu.edu/; East Lansing, MI). Raw read quality was assessed with FastQC (http:// www.bioinformatics.babraham.ac.uk/ projects/fastqc/). Sequences were filtered and trimmed based on quality metrics and adapter sequences were removed with TrimmomaticPE [68]. The TrimmomaticPE options employed included ILLUMINACLIP, SLIDINGWINDOW:5:20, LEADING:5, TRAILING:5, and HEADCROP:10-14. TrimmomaticPE outputs sequences into paired-end and single-end files requiring no further mate-specific filtering. Cleaned reads were reassessed with FastQC for quality visualization to ensure no further filtering was required.

\section{De novo assembly and expression analysis}

Reads meeting quality standards were de novo assembled using the Velvet (version 1.1.07) and Oases (version $0.2 .08)$ packages $[69,70]$. K-mer lengths of $55,57,59$, $61,63,65,67$, and 69 were tested and metrics collected for each assembly. Criterion including the total number of transcripts produced, Velvet N50 length, average transcript length, and average transcript read coverage were considered. A k-mer of 61 was selected representing the best balance of metrics for all assemblies. Two de novo assemblies were completed for each Petunia transcriptome to reduce redundancy and limit the number of raw reads confounding assemblies. Reads from the callus, shoot apex and seedling libraries composed the first de novo assembly. Resulting transcripts were concatenated into a single pseudomolecule or 'artificial chromosome one' to serve as our core tissue reference transcriptome. Reads from the trichome and flower libraries were then 
mapped to artificial chromosome one with Bowtie (version 1.0.0) and TopHat (version 1.4.1) [46]. Reads aligning to the artificial chromosome were discarded to eliminate redundancy of data already assembled. All unmapped reads from the flower and trichome libraries were then combined with the original callus, shoot apex, and seedling reads in a second de novo transcriptome assembly [42]; Supplemental Fig. 1). Transcripts from the second and final assembly were filtered for low complexity. Representative transcripts, comprised of the longest isoform were extracted from the final assemblies. Completeness of the assemblies was assessed by mapping all reads from each tissue library back to their respective de novo assembly (per species) individually with Bowtie and TopHat [46]. CEGMA or Core Eukaryotic Genes Mapping Approach [43], a pipeline used to accurately annotate core genes in eukaryotic genomes, was also searched to determine the quality and completeness of the assemblies. Reads from each tissue library were mapped back to the de novo assembly with Bowtie and TopHat. Estimation of transcript abundance was then completed with Cufflinks [71, 72]. Cufflinks reports expression abundance in Fragments Per Kilobase of exon model per Million fragments mapped, or FPKM. Values consider the number of reads supporting each model and counts are normalized.

\section{Functional annotation and identification of orthologous and paralogous gene families}

Putative functions were assigned using BLASTX queries against the Arabidopsis thaliana protein database (TAIR10; http://www.arabidopsis.org/), UniRef100 (UniProt Knowledgebase Reference Clusters; release 63; http:// www.uniprot.org/), RefSeq protein database (NCBI Reference Sequence Database; www.ncbi.nlm.nih.gov/refseq/), Pfam domains (version 27.0; pfam.xfam.org), and the NCBI non-redundant protein database (nr; www.ncbi.nlm.nih.gov/protein). BLASTX queries against all databases were searched using an e-value cutoff of $1 \mathrm{e}-5$ and allowing a single hit. Hits were then processed accepting only those with $>30 \%$ coverage and $>70 \%$ identity to the subject. A total of $126 P$. axillaris, $12 P$. integrifolia, and two $P$. exserta representative transcripts were removed as suspected contamination based on their annotation. Functional annotation of the transcripts was also queried using web-based FastAnnotator [73]. FastAnnotator facilitates the integration of the annotation tools Blast2GO, PRIAM, and RPS BLAST, providing gene ontology classifications (GO), enzyme and domain identification. Transcripts were translated in batch using OrfPredictor [74] and orthologous and paralogous proteins in the three species were assigned with OrthoMCL v2.0.2 with default parameters and an e-value cut-off of 1e-10 [50].

\section{SNP Identification}

Sequence reads from each tissue type were pooled for each species and mapped to the Petunia axillaris transcriptome using the Burrows-Wheeler Aligner (BWA) program [75] with default values. Duplicate reads were removed after the initial alignment, to eliminate reads that mapped to the same position of the transcriptome. Duplicate removal was performed for the aligned reads using picardTools/1.89 (broadinstitute.github.io/picard). The subsequent local realignment to correct misalignments due to the presence of indels was performed using the Genome Analysis Toolkit (GATK) [51] with the IndelRealigner function. Bam files for each genotype were compressed using ReduceReads from GATK. The initial variant calling was performed by HaplotypeCaller from GATK with a Phred-scaled confidence threshold of 30. After the initial variant calling, SNPs were filtered based on several criteria. The first round of SNP filtering employed the following steps: exclude three SNPs within $100 \mathrm{bp}$; filter out variants with zero mapping quality constituting more than $10 \%$ of all reads at that site; exclude SNPs covered only by sequences on the same strand with an FS value (Phred-scaled p-value using Fisher's exact test to detect strand bias) $>60$; exclude SNPs with a minor allele frequency $<0.01$; and exclude SNPs with low QD (quality by depth), low quality $(<11)$, and low total depth of coverage $(<11)$ by the default parameters recommended from GATK best practice with the VariantFiltration tool $[53,76]$. The second round of SNP filtering was performed by custom Python (Python 2.7.8) [77] scripts with the following criteria: SNPs within the beginning and end $30 \mathrm{bp}$ of the reference transcripts were excluded; SNPs were selected with at least two genotypes having a depth of coverage greater than 10; exclude loci with a heterozygous genotype call in Petunia axillaris. The intron-exon boundaries were predicted by aligning the Petunia axillaris transcripts to the draft Petunia axillaris genome scaffold sequences with GMAP [78]. SNPs within 30 bp of the exon-intron boundary region were then filtered out. Indels were not called because alternative splicing may impede reliable indel discovery. A custom Python script was used to extract the 5囚-UTR and 3\-UTR and to calculate the distribution of SNPs in these regions. Depth of coverage was calculated by BEDTools [79]. Annotated unigenes containing SNPs were visualized by BGI WEGO (web gene ontology annotation plotting) (http://wego.genomics.org.cn/cgi-bin/wego/index.pl). KEGG pathways were assigned to unigenes containing SNPs using the online KEGG Automatic Annotation Server (KAAS) (http://www.genome.jp/tools/kaas/) [80]. KEGG Orthology (KO) assignment was applied using Bi-directional Best Hit (BBH) method with plant organisms (Arabidopsis thaliana (thale cress), Arabidopsis lyrata (lyrate 
rockcress), Theobroma cacao (cacao), Glycine max (soybean), Fragaria vesca (woodland strawberry), Vitis vinifera (wine grape), Solanum lycopersicum (tomato), and Oryza sativa L. ssp. japonica (Japanese rice) (RefSeq)) as references.

\section{SNP validation}

A total of 55 SNPs were selected for validation. Primers were designed by first choosing SNPs where at least two species exhibited polymorphism. Unigenes with exons greater than $600 \mathrm{bp}$ were then selected, SNPs or alleles in the sequences were masked using IUBI./IUPAC nucleotide acid code. Batch Primer3 (http://probes.pw.us da.gov/cgi-bin/batchprimer3/batchprimer3.cgi) was used for primer design using the "SNP flanking primers design" option. The expected amplicon sizes ranged from 250 to $350 \mathrm{bp}$, with primer sizes ranging from 18 to $27 \mathrm{bp}$, and primer $\mathrm{T}_{\mathrm{m}}$ ranging from 57 to $63{ }^{\circ} \mathrm{C}$. Genomic DNA was extracted using the DNeasy plant mini kit (QIAGEN, Valencia, CA, USA). PCR amplification of genomic DNA was carried out in a $10 \mu$ reaction containing $1 \times$ PCR buffer, $0.2 \mu \mathrm{l} 10 \mu \mathrm{M}$ dNTPs, $0.6 \mathrm{U}$ of DNA polymerase, and $5 \mathrm{ng}$ template DNA. The following PCR profile was used: an initial denaturation at $95^{\circ} \mathrm{C}$ for $30 \mathrm{~s}$, followed by 11 cycles of $95^{\circ} \mathrm{C}$ for $30 \mathrm{~s}, 65^{\circ} \mathrm{C}$ to $55^{\circ} \mathrm{C}$ for $30 \mathrm{~s}$, with a decrease of $1{ }^{\circ} \mathrm{C}$ per cycle, and $72{ }^{\circ} \mathrm{C}$ for $30 \mathrm{~s}$, followed 30 cycles of $95{ }^{\circ} \mathrm{C}$ for $30 \mathrm{~s}, 58{ }^{\circ} \mathrm{C}$ for $30 \mathrm{~s}$, and $72{ }^{\circ} \mathrm{C}$ for $30 \mathrm{~s}$, followed by a final extension at $72{ }^{\circ} \mathrm{C}$ for $5 \mathrm{~min}$. Amplicons were purified by Agencourt AMPure XP beads (Beckman Coulter, Pasadena, CA) and quantified by BioDrop Duo (Isogen Life Science, the Netherlands). Sanger sequencing of the amplicons was performed on an ABI 3730xl platform (Life Technologies, Carlsbad, CA) at the Michigan State University Genomics Core Facility. Sequences amplified from the same primer set were initially aligned with CLC Sequence Viewer 6.9.1 (CLC Bio, Valencia, CA, USA) for preliminary SNP verification. Sequence chromatograms were visualized by Seq Scanner 2 (Life Technologies, Carlsbad, CA) for further SNP confirmation.

\section{CAPS and SSR marker design}

SNP markers were transformed into CAPS markers using the following pipeline. First, the SNP output was transformed into VCF (variant call format) format. Then, by using the previous GMAP output as reference, a new VCF file was generated by transforming the current SNP locations and genotypes to corresponding SNP locations and variant calls in the $P$. axillaris draft genome scaffolds by a custom Python script. Primers were designed with the galaxy-pcr-markers pipeline (https://github. com/cfljam/galaxy-pcr-markers) with the modification of producing only one pair of primers, minimum amplicon size of $200 \mathrm{bp}$ and maximum amplicon size of $400 \mathrm{bp}$. A total of 20 commonly used restriction enzymes: $A l u \mathrm{I}$, ApaI, BamHI, BbrPI, BfrI, ClaI, DdeI, DpnII, DraI, EcoRI, HaeIII, HincII, HinfI, HpaI, PvuII, RsaI, SacI, Sau3AI, SmaI, and TaqI were selected for the pipeline. The unigene dataset were used for SSR identification with MISA (microsatellite identification tool) (http://pgrc.ipk-gatersleben.de/misa/). The SSR identification criteria were $6,5,5$, 5, 5 repeats for di-, tri-, tetra-, penta-, and hexanucleotides, respectively. Primer pairs were designed from primer3 core (Primer3 2.3.6) (http://primer3.sourceforge.net/releases.php). Primer parameters were: designated amplicon size 100-280 bp, primer annealing temperature 55 to $65{ }^{\circ} \mathrm{C}$, primer length 18 to $28 \mathrm{bp}$, and GC content from 45 to $55 \%$. Unigenes with homology to known genes involved in specifying plant development rate [16-18, 20, 25] were searched for possible CAPS markers from the entire CAPS output. The CAPS markers were used to genotype the three species by following the previous PCR protocol. CAPS markers were digested by the above mentioned restriction enzymes (New England Biolabs, Beverly, MA) and separated on $2 \%$ NuSieve GTG agarose (Lonza, Basel, Switzerland) plus $1 \%$ UltraPure agarose (Life Technologies, Carlsbad, CA) gel with $100 \mathrm{~V}$ for $2 \mathrm{~h}$ at room temperature.

\section{Candidate gene mapping and QTL mapping of development rate in an interspecific hybrid Petunia population}

An interspecific hybrid $F_{2}$ population developed from a cross between $P$. integrifolia and $P$. axillaris containing 164 individuals was used to identify QTL for development rate. Population development, the measurement of development rate, and the SSR-based genetic linkage map were reported previously [11]. CAPS markers developed from $P$. axillaris plastochron-related transcripts based on SNPs between $P$. integrifolia and $P$. axillaris were used to screen the $\mathrm{F}_{2}$ population. If no CAPS were previously designed from our SNP set, CAPS markers were manually designed with CAPS Designer (http:// solgenomics.net/tools/caps_designer/caps_input.pl) and Primer3Plus (http://www.bioinformatics.nl/cgi-bin/pri mer3plus/primer3plus.cgi/). The updated genetic linkage map was generated with JoinMap 4.0 [81] with the Kosambi mapping function [82]. The recombination threshold was set to 0.3 and the logarithmic odds (LOD) score was set to 5 . The locus order was calculated by the regression module of JoinMap4. Linkage group numbers were assigned according to the previous study [11]. QTL mapping was conducted with MapQTL 6 [83]. A permutation test with 1000 permutations was used to establish the LOD significance threshold. The initial QTL mapping was performed by interval mapping (a single-QTL model). Multiple QTL model mapping (MQM) was then used to reduce the background 
noise. Significant QTL and regions were graphically visualized using MapChart 2.1 [84].

\section{Accession numbers}

Sequences are available under Genbank/EMBL/DDBJ BioProject numbers PRJNA262254, PRJNA262142, and PRJNA261953. These include SRA (Sequence Read Archive) runs for P. axillaris: SRR1585615, SRR1585635, SRR1585830, and SRR1585954-1585955; P. exserta: SRR1586492-1586494, SRR1586500, and SRR1586504; P. integrifolia: SRR1587109, SRR1587150-1587151, and SRR1587153-1587154. Transcriptome Shotgun Assembly projects were also deposited under accessions GBRU00 000000 ( $P$. axillaris), GBRT00000000 (P. exserta), and GBRV00000000 ( $P$. integrifolia). The versions described in this paper are the first versions of these assemblies (GBRU01000000, GBRT01000000, GBRV01000000). The SNPs are available at NCBI dbSNP under accession numbers ss1750993386 - ss1751108187.

\section{Additional files}

Additional file 1: Figure S1. Work flow used for assemblies allowing incorporation of reads from all 5 tissue libraries while removing redundancy. Figure S2. Distributions of the number of isoforms for each transcript. Figure S3. SNP discovery pipeline with reads aligned to P. axillaris reference transcriptome assembly. Figure S4. Gene Ontology (GO) categories of the unigenes and unigenes with SNPs. Figure S5. Gene Ontology (GO) categories of the unigenes with more than ten SNPs. Distribution of the GO categories assigned to the unigenes were annotated in three categories: cellular components, molecular functions, and biological processes. Table S1. Primer sequences used for SNP validation by Sanger sequencing. Table S2. Primer sequences for CAPS markers developed from plastochron-related genes. (DOCX $782 \mathrm{~kb}$ )

Additional file 2: Supplemental Dataset 1. Petunia unigene locus identifiers, expression levels, annotation and sequences. (XLSX 11680 kb)

Additional file 3: Supplemental Dataset 2. OrthoMCL clusters across three Petunia species. (XLSM 1433 kb)

Additional file 4: Supplemental Dataset 3. Gene Ontology annotations of representative transcripts. (XLS $16720 \mathrm{~kb}$ )

Additional file 5: Supplemental Dataset 4. CAPS markers developed from SNPS. (XLSX $1499 \mathrm{~kb}$ )

Additional file 6: Supplemental Dataset 5. SSR primers derived from P. axillaris transcriptome. (XLSX $414 \mathrm{~kb}$ )

Additional file 7: Supplemental Dataset 6. SSR validation results. (XLSX $13 \mathrm{~kb})$

\section{Competing interests}

The authors report no competing interests in the funding or interpretation of this study.

\section{Authors' contributions}

RMW and CSB designed and coordinated the study and assisted with data analysis. YG performed the molecular marker discovery and analysis, and mapping of the plastochron-related transcripts. KEW-R. conducted the bioinformatics associated with transcriptome assemblies and analysis. VAV collected and prepared samples for sequencing, and assisted with the assembly and analysis of the transcriptomes. KEW-R, YG, CSB and RMW wrote the manuscript. All authors read and approved the manuscript.

\section{Acknowledgements}

Funding was provided by USDA Specialty Crops Research Initiative Award number 2011-51181-30666 to R.M.W. and C.S.B. and National Science Foundation award number IOS-1025636 and a Michigan State University (MSU) Foundation Strategic Partnership Grant to C.S.B. R.M.W. and C.S.B. are supported in part by Michigan AgBioResearch and through USDA National Institute of Food and Agriculture, Hatch project numbers MICL02121 and MICL02265. The authors would like to thank the staff at the Michigan State University Research Technology Support Facility for TruSeq library preparation and transcriptome sequencing. We would also like to thank Dr. Jin-Ho Kang for collection of trichomes and Stephanie Rett for her technical support and assistance with SNP validation.

Received: 5 May 2015 Accepted: 16 September 2015

Published online: 24 September 2015

\section{References}

1. Reck-Kortmann M, Silva-Arias GA, Segatto ALA, Mader C, Bonatto SL, de Freitas LB. Multilocus phylogeny reconstruction: New insights into the evolutionary history of the genus Petunia. Mol Phylogenet Evol. 2014,81:19-28.

2. Stehmann JR, Lorenz-Lemke AP, Freitas LB, Semir J. The genus Petunia. In: Gerats T, Strommer J, editors. Petunia Evolutionary, Developmental and Physiological Genetics. New York: Springer; 2009. p. 1-28.

3. Morita Y, Saito R, Ban Y, Tanikawa N, Kuchitsu K, Ando T, et al. Tandemly arranged chalcone synthase $\mathrm{A}$ genes contribute to the spatially regulated expression of siRNA and the natural bicolor floral phenotype in Petunia hybrida. Plant J. 2012;70(5):739-49.

4. Kelly RO, Deng ZA, Harbaugh BK. Evaluation of 125 petunia cultivars as bedding plants and establishment of class standards. Horttechnology. 2007;17(3):386-96.

5. Kroon J, Souer E, Degraaff A, Xue YB, Mol J, Koes R. Cloning and structural analysis of the anthocyanin pigmentation locus Rt of Petunia hybrida: Characterization of insertion sequences in two mutant alleles. Plant J. 1994;5(1):69-80.

6. Holton TA, Brugliera F, Lester DR, Tanaka Y, Hyland CD, Menting JGT, et al. Cloning and expression of cytochrome P450 genes controlling flower color. Nature. 1993;366(6452):276-9.

7. Quattrocchio F, Wing J, van der Woude K, Souer E, de Vetten N, Mol J, et al. Molecular analysis of the anthocyanin2 gene of petunia and its role in the evolution of flower color. Plant Cell. 1999;11(8):1433-44

8. Bartok JW. Energy conservation for commercial greenhouses. New York: Cooperative Extension, Ithaca; 2001

9. Blanchard MG, Runkle ES. The influence of day and night temperature fluctuations on growth and flowering of annual bedding plants and greenhouse heating cost predictions. Hortscience. 2011;46(4):599-603.

10. Warner RM, Walworth AE. Quantitative inheritance of crop timing traits in interspecific hybrid Petunia populations and interactions with crop quality parameters. J Hered. 2010;101(3):308-16.

11. Vallejo VA, Tychonievich J, Lin W-K, Wangchu L, Barry CS, Warner RM. Identification of QTL for crop timing and quality traits in an interspecific Petunia population. Molecular Breeding. 2015, 35(2):doi:10.1007/s1103211015-10218-11034.

12. Werner T, Motyka V, Strnad M, Schmulling T. Regulation of plant growth by cytokinin. Proc Natl Acad Sci U S A. 2001;98(18):10487-92

13. Lohmann D, Stacey N, Breuninger H, Jikumaru $Y$, Muller D, Sicard A, et al. SLOW MOTION is required for within-plant auxin homeostasis and normal timing of lateral organ initiation at the shoot meristem in Arabidopsis. Plant Cell. 2010;22(2):335-48.

14. Chen MK, Wilson RL, Palme K, Ditengou FA, Shpak ED. ERECTA family genes regulate auxin transport in the shoot apical meristem and forming leaf primordia. Plant Physiol. 2013;162(4):1978-91.

15. Guenot B, Bayer E, Kierzkowski D, Smith RS, Mandel T, Zadnikova P, et al. PIN1-independent leaf initiation in Arabidopsis. Plant Physiol. 2012;159(4):1501-10

16. Miyoshi K, Ahn BO, Kawakatsu T, Ito Y, Itoh Jl, Nagato Y, et al. PLASTOCHRON1, a timekeeper of leaf initiation in rice, encodes cytochrome P450. Proc Natl Acad Sci U S A. 2004;101(3):875-80.

17. Kawakatsu T, Itoh J, Miyoshi K, Kurata N, Alvarez N, Veit B, et al. PLASTOCHRON2 regulates leaf initiation and maturation in rice. Plant Cell. 2006;18(3):612-25. 
18. Wang JW, Schwab R, Czech B, Mica E, Weigel D. Dual effects of miR156targeted SPL genes and CYP78A5/KLUH on plastochron length and organ size in Arabidopsis thaliana. Plant Cell. 2008;20(5):1231-43.

19. Grigg SP, Canales C, Hay A, Tsiantis M. SERRATE coordinates shoot meristem function and leaf axial patterning in Arabidopsis. Nature. 2005;437(7061):1022-6

20. Prigge MJ, Wagner DR. The Arabidopsis SERRATE gene encodes a zinc-finger protein required for normal shoot development. Plant Cell. 2001;13(6):1263-79.

21. Chaudhury AM, Letham S, Craig S, Dennis ES. amp1 - a mutant with high cytokinin levels and altered embryonic pattern, faster vegetative growth, constitutive photomorphogenesis and precocious flowering. Plant J. 1993:4(6):907-16.

22. Helliwell CA, Chin-Atkins AN, Wilson IW, Chapple R, Dennis ES, Chaudhury A. The Arabidopsis AMP1 gene encodes a putative glutamate carboxypeptidase. Plant Cell. 2001;13(9):2115-25.

23. Li SB, Liu L, Zhuang XH, Yu Y, Liu XG, Cui X, et al. MicroRNAs inhibit the translation of target mRNAs on the endoplasmic reticulum in Arabidopsis. Cell. 2013;153(3):562-74.

24. Evans MMS, Poethig RS. The viviparous 8 mutation delays vegetative phase change and accelerates the rate of seedling growth in maize. Plant J. 1997;12(4):769-79.

25. Kawakatsu T, Taramino G, Itoh Jl, Allen J, Sato Y, Hong SK, et al. PLASTOCHRON3/GOLIATH encodes a glutamate carboxypeptidase required for proper development in rice. Plant J. 2009;58(6):1028-40.

26. Wang Z, Gerstein M, Snyder M. RNA-Seq: a revolutionary tool for transcriptomics. Nat Rev Genet. 2009;10(1):57-63.

27. Ozsolak F, Milos PM. RNA sequencing: advances, challenges and opportunities. Nat Rev Genet. 2011;12(2):87-98.

28. Schilmiller AL, Miner DP, Larson M, McDowell E, Gang DR, Wilkerson C, et al. Studies of a biochemical factory: tomato trichome deep expressed sequence tag sequencing and proteomics. Plant Physiol. 2010;153(3):1212-23.

29. Varshney RK, Nayak SN, May GD, Jackson SA. Next-generation sequencing technologies and their implications for crop genetics and breeding. Trends Biotechnol. 2009:27(9):522-30

30. Hamilton JP, Sim SC, Stoffel K, Van Deynze A, Buell CR, Francis DM. Single nucleotide polymorphism discovery in cultivated tomato via sequencing by synthesis. Plant Genome. 2012;5(1):17-29.

31. Barbazuk WB, Emrich SJ, Chen HD, Li L, Schnable PS. SNP discovery via 454 transcriptome sequencing. Plant J. 2007:51(5):910-8.

32. Hirsch CN, Foerster JM, Johnson JM, Sekhon RS, Muttoni G, Vaillancourt B, et al. Insights into the maize pan-genome and pan-transcriptome. Plant Cell. 2014;26(1):121-35.

33. Bedewitz MA, Gongora-Castillo E, Uebler JB, Gonzales-Vigil E, Wiegert-Rininger $\mathrm{KE}$, Childs KL, et al. A root-expressed L-phenylalanine: 4-hydroxyphenylpyruvate aminotransferase is required for tropane alkaloid biosynthesis in Atropa belladonna. Plant Cell. 2014;26(9):3745-62

34. Galliot C, Hoballah ME, Kuhlemeier C, Stuurman J. Genetics of flower size and nectar volume in Petunia pollination syndromes. Planta. 2006;225(1):203-12.

35. Klahre U, Gurba A, Hermann K, Saxenhofer M, Bossolini E, Guerin PM, et al. Pollinator choice in petunia depends on two major genetic loci for floral scent production. Curr Biol. 2011;21(9):730-9.

36. Bossolini E, Klahre U, Brandenburg A, Reinhardt D, Kuhlemeier C. High resolution linkage maps of the model organism Petunia reveal substantial synteny decay with the related genome of tomato. Genome. 2011:54(4):327-40.

37. Strommer J, Gerats AGM, Sanago M, Molnar SJ. A gene-based RFLP map of Petunia. Theor Appl Genet. 2000;100(6):899-905.

38. Strommer J, Peters J, Zethof J, De Keukeleire P, Gerats T. AFLP maps of Petunia hybrida: building maps when markers cluster. Theor Appl Genet. 2002;105(6-7):1000-9.

39. Villarino GH, Bombarely A, Giovannoni JJ, Scanlon MJ, Mattson NS Transcriptomic analysis of Petunia hybrida in response to salt stress using high throughput RNA sequencing. Plos One. 2014;9(4):e94651.

40. Williams JS, Der JP, dePamphilis CW, Kao TH. Transcriptome analysis reveals the same 17 S-locus F-box genes in two haplotypes of the selfincompatibility locus of Petunia inflata. Plant Cell. 2014;26(7):2873-88

41. Zenoni S, D'Agostino N, Tornielli GB, Quattrocchio F, Chiusano ML, Koes R, et al. Revealing impaired pathways in the an11 mutant by high-throughput characterization of Petunia axillaris and Petunia inflata transcriptomes. Plant J. 2011;68(1):11-27.

42. Gongora-Castillo E, Fedewa G, Yeo Y, Chappell J, DellaPenna D, Buell CR. Genomic approaches for interrogating the biochemistry of medicinal plant species. Method Enzymol. 2012;517:139-59.

43. Parra G, Bradnam K, Korf I. CEGMA: a pipeline to accurately annotate core genes in eukaryotic genomes. Bioinformatics. 2007;23(9):1061-7.

44. Zerbe P, Hamberger B, Yuen MMS, Chiang A, Sandhu HK, Madilao LL, et al. Gene discovery of modular diterpene metabolism in nonmodel systems. Plant Physiol. 2013;162(2):1073-91.

45. Nakasugi K, Crowhurst RN, Bally J, Wood CC, Hellens RP, Waterhouse PM. De novo transcriptome sequence assembly and analysis of RNA silencing genes of Nicotiana benthamiana. Plos One. 2013;8(3):e59534.

46. Trapnell C, Pachter L, Salzberg SL. TopHat: discovering splice junctions with RNA-Seq. Bioinformatics. 2009;25(9):1105-11.

47. Mather K. Specific differences in Petunia I. Incompatibility. J Genet 1943:45(3):215-35

48. Garg R, Patel RK, Tyagi AK, Jain M. De novo assembly of chickpea transcriptome using short reads for gene discovery and marker identification. DNA Res. 2011;18(1):53-63.

49. Yates SA, Swain MT, Hegarty MJ, Chernukin I, Lowe M, Allison GG, et al. De novo assembly of red clover transcriptome based on RNA-Seq data provides insight into drought response, gene discovery and marker identification. BMC Genomics. 2014;15:453.

50. Li L, Stoeckert CJ, Roos DS. OrthoMCL: Identification of ortholog groups for eukaryotic genomes. Genome Res. 2003;13(9):2178-89.

51. McKenna A, Hanna M, Banks E, Sivachenko A, Cibulskis K, Kernytsky A, et al. The genome analysis toolkit: a MapReduce framework for analyzing next-generation DNA sequencing data. Genome Res. 2010;20(9):1297-303.

52. Quinn EM, Cormican P, Kenny EM, Hill M, Anney R, Gill M, et al. Development of strategies for SNP detection in RNA-seq data: application to lymphoblastoid cell lines and evaluation using 1000 genomes data. Plos One. 2013;8(3):e58815.

53. DePristo MA, Banks E, Poplin R, Garimella KV, Maguire JR, Hartl C, et al. A framework for variation discovery and genotyping using next-generation DNA sequencing data. Nat Genet. 2011:43(5):491-8.

54. Niu S-H, Li Z-X, Yuan H-W, Chen X-Y, Li Y, Li W. Transcriptome characterisation of Pinus tabuliformis and evolution of genes in the Pinus phylogeny. BMC Genomics. 2013;14:263.

55. Miguel Blanca J, Canizares J, Ziarsolo P, Esteras C, Mir G, Nuez F, et al. Melon transcriptome characterization: Simple sequence repeats and single nucleotide polymorphisms discovery for high throughput genotyping across the species. Plant Genome. 2011;4(2):118-31

56. Coulondre C, Miller JH, Farabaugh PJ, Gilbert W. Molecular basis of base substitution hotspots in Escherichia coli. Nature. 1978;274(5673):775-80.

57. Keller I, Bensasson D, Nichols R. Transition-transversion bias is not universal: a counter example from grasshopper pseudogenes. PLoS Genet. 2007;3(2):e22.

58. Brousseau L, Tinaut A, Duret C, Lang T, Garnier-Gere P, Scotti I. High-throughput transcriptome sequencing and preliminary functional analysis in four Neotropical tree species. BMC Genomics. 2014;15:238.

59. Chen J, Uebbing S, Gyllenstrand N, Lagercrantz U, Lascoux M, Kallman T. Sequencing of the needle transcriptome from Norway spruce (Picea abies Karst L.) reveals lower substitution rates, but similar selective constraints in gymnosperms and angiosperms. BMC Genomics. 2012;13:589.

60. Duarte J, Riviere N, Baranger A, Aubert G, Burstin J, Cornet L, et al. Transcriptome sequencing for high throughput SNP development and genetic mapping in Pea. BMC Genomics. 2014;15:126.

61. Howe GT, Yu J, Knaus B, Cronn R, Kolpak S, Dolan P, et al. A SNP resource for Douglas-fir: de novo transcriptome assembly and SNP detection and validation. BMC Genomics. 2013;14:137.

62. Hamilton JP, Hansey CN, Whitty BR, Stoffel K, Massa AN, Van Deynze A, et al. Single nucleotide polymorphism discovery in elite north american potato germplasm. BMC Genomics. 2011;12:302.

63. Raji AA, Anderson JV, Kolade OA, Ugwu CD. Gene-based microsatellites for cassava (Manihot esculenta): prevalence, polymorphisms, and cross-taxa utility. BMC Plant Biol. 2009:9:118.

64. Poncet V, Rondeau M, Tranchant C, Cayrel A, Hamon S. SSR mining in coffee tree EST databases: potential use of EST-SSRs as markers for the Coffea genus. Mol Genet Genomics. 2006;276(5):436-49. 
65. Guo Y, Khanal S, Tang S, Bowers J, Heesacker A. Comparative mapping in intraspecific populations uncovers a high degree of macrosynteny between A-and B-genome diploid species of peanut. BMC Genomics. 2012;13:608.

66. Tychonievich J, Wangchu L, Barry C, Warner RM. Utilizing wild species for marker-assisted selection of crop timing and quality traits in Petunia. Acta Hortic. 2013;1000:465-9.

67. Zubko E, Adams CJ, Machaekova I, Malbeck J, Scollan C, Meyer P. Activation tagging identifies a gene from Petunia hybrida responsible for the production of active cytokinins in plants. Plant J. 2002;29(6):797-808.

68. Bolger AM, Lohse M, Usadel B. Trimmomatic: a flexible trimmer for Illumina sequence data. Bioinformatics. 2014;30(15):2114-20.

69. Zerbino DR, Birney E. Velvet: algorithms for de novo short read assembly using de Bruijn graphs. Genome Res. 2008;18(5):821-9.

70. Schulz MH, Zerbino DR, Vingron M, Birney E. Oases: robust de novo RNA-seq assembly across the dynamic range of expression levels. Bioinformatics. 2012;28(8):1086-92.

71. Trapnell C, Roberts A, Goff L, Pertea G, Kim D, Kelley DR, et al. Differential gene and transcript expression analysis of RNA-seq experiments with TopHat and Cufflinks. Nat Protoc. 2012;7(3):562-78.

72. Trapnell C, Williams BA, Pertea G, Mortazavi A, Kwan G, van Baren MJ, et al. Transcript assembly and quantification by RNA-Seq reveals unannotated transcripts and isoform switching during cell differentiation. Nat Biotech. 2010;28(5):511-5.

73. Chen TW, Gan RCR, Wu TH, Huang PJ, Lee CY, Chen YYM, et al. FastAnnotator- an efficient transcript annotation web tool. BMC Genomics. 2012;13(7):S9.

74. Min XJ, Butler G, Storms R, Tsang A. OrfPredictor: predicting protein-coding regions in EST-derived sequences. Nucleic Acids Res. 2005;33:W677-80.

75. Li H, Durbin R. Fast and accurate short read alignment with BurrowsWheeler transform. Bioinformatics. 2009;25(14):1754-60.

76. Van der Auwera GA, Carneiro MO, Hartl C, Poplin R, del Angel G, Levy-Moonshine A, Jordan T, Shakir K, Roazen D, Thibault J et al.: From FastQ data to high-confidence variant calls: The genome analysis toolkit best practices pipeline. Current Protocols in Bioinformatics. 2013:43:11.10.1-11.10-33.

77. van Rossum $\mathrm{G}$, de Boer J. Interactively testing remote servers using the Python programming language. CWI Quarterly. 1991;4(4):283-303.

78. Wu TD, Watanabe CK. GMAP: a genomic mapping and alignment program for mRNA and EST sequences. Bioinformatics. 2005;21(9):1859-75.

79. Quinlan AR, Hall IM. BEDTools: a flexible suite of utilities for comparing genomic features. Bioinformatics. 2010;26(6):841-2.

80. Moriya Y, Itoh M, Okuda S, Yoshizawa AC, Kanehisa M. KAAS: an automatic genome annotation and pathway reconstruction server. Nucleic Acids Res. 2007:35:W182-5.

81. Van Ooijen JW. JoinMap ${ }^{\circledR}$ 4, Software for the calculation of genetic linkage maps in experimental populations. Wageningen, Netherlands: Kyazma BV; 2006.

82. Kosambi DD. The estimation of map distances from recombination values. Ann Eugenics. 1944;12:172-5.

83. Van Ooijen JW. MapQTL $6^{\oplus}$, Software for the mapping of quantitative trait loci in experimental populations of diploid species. Wageningen, Netherlands: Kyazma BV; 2009.

84. Voorrips RE. MapChart: software for the graphical presentation of linkage maps and QTLs. J Hered. 2002;93(1):77-8.

\section{Submit your next manuscript to BioMed Central and take full advantage of:}

- Convenient online submission

- Thorough peer review

- No space constraints or color figure charges

- Immediate publication on acceptance

- Inclusion in PubMed, CAS, Scopus and Google Scholar

- Research which is freely available for redistribution

Submit your manuscript at www.biomedcentral.com/submit 